\title{
PLANTEAMIENTO DE FACTORES DE AMPLIFICACIÓN DE RESISTENCIA PARA ESTRUCTURAS CON ASIMETRÍA EN FLUENCIA
}

\author{
Amador Terán Gilmore y Danny Arroyo Espinoza ${ }^{(1)}$
}

\begin{abstract}
RESUMEN
Algunas estructuras exhiben asimetría en la fuerza de fluencia que desarrollan en sentidos opuestos. Para evitar un desempeño sísmico insatisfactorio de estructuras con asimetría en fluencia, se requiere diseñarlas para un mayor nivel de resistencia lateral que el contemplado para estructuras simétricas. Este artículo plantea expresiones para estimar factores de amplificación de resistencia que permitan establecer el espectro de diseño para sistemas con asimetría en fluencia. Las expresiones toman en cuenta el grado de asimetría en fluencia y comportamiento histerético de la estructura, el tipo de terreno donde se desplanta, y la relación que guarda su periodo fundamental de vibración con respecto al periodo dominante del terreno.
\end{abstract}

\begin{abstract}
Some structures exhibit asymmetrical yielding when loaded in opposing directions. To prevent unsatisfactory seismic performance, structures with asymmetrical yielding need to be designed for a higher level of lateral strength than that contemplated for symmetrical structures. This paper formulates expressions to estimate strength amplification factors that can be used to establish design spectra for systems that exhibit asymmetrical yielding. The expressions take into consideration the level of asymmetrical yielding and hysteretic behavior of the structure, the type of soil where it is built, and the relation between its fundamental period of vibration and the dominant period of the soil.
\end{abstract}

\section{INTRODUCCIÓN}

En diversas estructuras ubicadas en suelo blando, como es el caso de un número importante de edificios construidos en la Zona del Lago de la Ciudad de México, se han observado desplomos importantes. Como consecuencia, estructuras originalmente simétricas exhiben comportamiento asimétrico ante cargas laterales. Esta asimetría, que también puede presentarse por otras razones, tal como la presencia de mezanines y apéndices y la asimetría de carga vertical, puede afectar significativamente el comportamiento sísmico de las estructuras.

Artículo recibido el 20 de julio de 2004 y aprobado para su publicación el 28 de marzo de 2005. Se aceptarán comentarios y/o discusiones hasta cinco meses después de su publicación.

(1) Universidad Autónoma Metropolitana - Azcapotzalco, Av. San Pablo \# 180, Colonia Reynosa Tamaulipas, Azcapotzalco, 02200 México,DF.tga@correo.azc.uam.mx, aresda@correo.azc.uam.mx 
Dada la importancia práctica de prevenir el comportamiento inadecuado de estructuras existentes que presentan un desplomo significativo, es conveniente que se discuta y cuantifique la relación que existe entre dicho desplomo y la asimetría en fluencia que produce. Un desplomo resulta en una reducción del cortante basal de fluencia $\left(V_{y}\right)$ en dirección de dicho desplomo, y en un incremento de $V_{y}$ en dirección contraria, de tal manera que una estructura originalmente simétrica exhibe direcciones "débil" y "fuerte". Conforme a lo mostrado en la fig. 1, si se desprecian los efectos de segundo orden un oscilador simple con desplomo puede idealizarse como un oscilador sin desplomo pero con asimetría en fluencia (Terán et al. 1998). En la fig. 1, $W$ y $c$ son el peso reactivo y el coeficiente sísmico, respectivamente, del oscilador, y $\alpha$ el ángulo de desplomo en radianes. $\Delta_{y}$ es el desplazamiento lateral de fluencia del oscilador sin desplomo, y $V_{y}^{+}$y $V_{y}^{-}$son, respectivamente, los cortantes basales de fluencia en la dirección del desplomo y en la dirección contraria al desplomo.

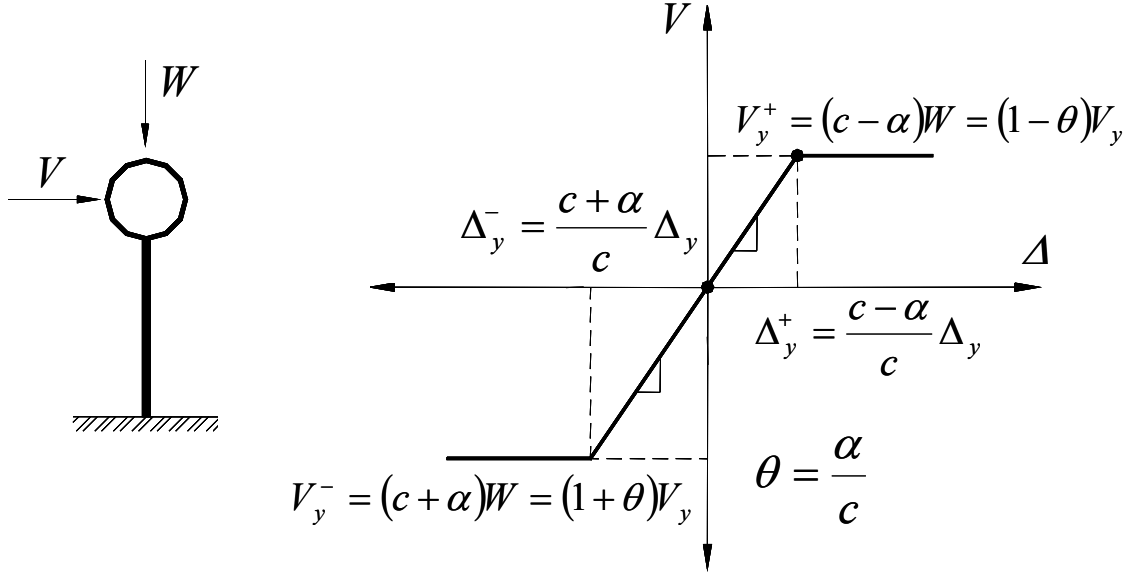

Figura 1. Efecto de una inclinación inicial $\alpha$ en el comportamiento monotónico de un oscilador

Terán-Gilmore et al. (2000) observan que el efecto de un desplomo dado $\alpha$ depende de la resistencia lateral de la estructura, de tal manera que es conveniente caracterizar numéricamente el nivel de asimetría en fluencia a través del parámetro $\theta$, definido conforme se muestra en la fig. 1 , como $\alpha / c$. Esto implica que una misma inclinación provoca diferente grado de asimetría en fluencia en sistemas con diferente resistencia. En caso de que la asimetría en fluencia se deba a otras razones, el grado de asimetría puede establecerse como:

$$
\theta=\frac{V_{y}^{-}-V_{y}^{+}}{V_{y}^{-}+V_{y}^{+}}
$$

donde $V_{y}^{+}$y $V_{y}^{-}$son, respectivamente, los cortantes basales de fluencia en las dirección débil y fuerte de la estructura.

Debido a la importancia que el efecto de la asimetría en fluencia puede llegar a tener en el desempeño sísmico de las estructuras, las Normas Técnicas Complementarias para Diseño por Sismo (NTCS) del Reglamento de Construcciones del D.F. incluyen requerimientos para el diseño de estructuras que exhiban este tipo de asimetría. Después de que en 1987 dichos requerimientos se 
introdujeron por primera vez a las NTCS, varios investigadores mexicanos han estudiado el efecto de la asimetría en fluencia en el desempeño de las estructuras sismorresistentes (Ruiz et al. 1989, 1990; Terán et al. 1993 y 1998, Terán-Gilmore et al. 2000). A pesar de que los resultados obtenidos hasta la fecha indican que la actual propuesta incluida en las NTCS para el diseño de estructuras con asimetría en fluencia no contempla el efecto de varias variables relevantes para su desempeño sísmico, esta se ha mantenido sin cambios desde la fecha de su incorporación al reglamento. En particular, una normatividad que no contemple el comportamiento histerético y las características dinámicas de la estructura, y el contenido de frecuencias de la excitación sísmica, no permitirá un diseño sísmico racional de estructuras con asimetría en fluencia.

Dado lo anterior, es de interés plantear propuestas racionales para el diseño de la resistencia lateral de estructuras con asimetría en fluencia, y que conformen una base sólida para el planteamiento de una normatividad que refleje de mejor manera la complejidad del efecto que la asimetría en fluencia tiene en el desempeño de las estructuras sismorresistentes.

\section{EVALUACIÓN DEL DESEMPEÑO SÍSMICO DE ESTRUCTURAS SISMORRESISTENTES}

El caso de mayor interés práctico asociado a la asimetría en fluencia, es el efecto que esta pueda tener en el desempeño sísmico de las estructuras para el estado límite de seguridad; en particular, en la posible influencia de dicha asimetría en el colapso o falla de estructuras durante sismos intensos. Actualmente, la posible falla de una estructura sismorresistente se evalúa a partir de su demanda máxima de ductilidad $\left(\mu_{\max }\right)$, que se define como el desplazamiento lateral máximo de la estructura durante la excitación sísmica normalizado por el desplazamiento lateral de fluencia. Dentro del contexto de la normatividad actual, el diseño contra colapso de una estructura consiste en proveerla con suficiente resistencia y rigidez lateral de manera que su $\mu_{\max }$ durante la excitación sísmica de diseño sea menor que la ductilidad última $\left(\mu_{u}\right)$ que alcanza cuando se le sujeta a un estado de desplazamiento lateral monotónicamente creciente (esto es, a carga unidireccional).

Investigaciones recientes han demostrado que, además de la demanda máxima de deformación, en el caso de suelos blandos las demandas acumuladas de deformación plástica pueden tener un efecto significativo en el nivel de daño estructural que exhiben las estructuras después de una excitación sísmica intensa (Terán y Jirsa 2003). Conforme a esto, es necesario contemplar criterios de evaluación estructural que consideren explícitamente las demandas acumuladas de deformación plástica durante la definición de factores de amplificación de resistencia para estructuras con asimetría en fluencia ubicadas en la Ciudad de México.

Una manera cuantitativa de tomar en cuenta las demandas acumuladas de deformación plástica es por medio de índices de daño, tal como el propuesto por Park y Ang (1985):

$$
D M I_{P A}=\frac{\mu_{\max }}{\mu_{u}}+\beta \frac{N E_{H \mu}}{\mu_{u}}
$$


donde $N E_{H \mu}$ es la energía plástica disipada durante el movimiento sísmico normalizada por el producto de la resistencia y deformación de fluencia de la estructura. $D M I_{P A}$ menor o igual que 0.4 puede interpretarse como daño reparable; de 0.4 a 1.0, como daño irreparable; y mayor o igual que 1.0, como falla. El trabajo realizado por varios investigadores sugiere que $\beta$ de 0.15 corresponde a sistemas que exhiben un comportamiento histerético razonablemente estable (Cosenza et al. 1993, Silva y López 2001); mientras que deben utilizarse valores de $\beta$ entre 0.2 y 0.4 para evaluar daño en sistemas que exhiben degradación considerable de resistencia y rigidez (Williams y Sexsmith 1997). Debe mencionarse que las calibraciones que del índice de Park y Ang han hecho varios investigadores presentan algunas inconsistencias, principalmente porque no existe una definición universalmente aceptada de lo que constituye la falla de un elemento. En la mayoría de los casos, se ha considerado como falla el estado que exhibe el elemento estructural cuando su resistencia se ha reducido por debajo de un porcentaje dado de la resistencia máxima que alcanza (del orden del 75 al 80\%).

Dentro del contexto de uso del índice de Park y Ang, $N E_{H \mu}$ es el parámetro que cuantifica la severidad de las demandas acumuladas de deformación plástica, y $\beta$ un parámetro que caracteriza la estabilidad del ciclo histerético de la estructura. Cabe aclarar que el parámetro $\beta$ utilizado en la ec. 2 es el mismo que utiliza el modelo de los tres parámetros, descrito posteriormente, para degradar la resistencia del modelo histerético.

La ec. 2 fue planteada originalmente para evaluar daño estructural en elementos de concreto reforzado. Sin embargo, investigaciones recientes han mostrado que, cuando la respuesta dinámica no lineal este dominada por el modo fundamental de vibración, es posible replantearla para evaluar el daño global en marcos momento-resistentes con y sin asimetría en fluencia (Terán-Gilmore et al. 2000, Terán 2002). Cuando $D M I_{P A}$ se utiliza para evaluar daño global en una estructura compleja a través del uso de un sistema equivalente de un grado de libertad (1GL), $\mu_{\max } \mathrm{y} \mu_{u}$ deben plantearse en función del desplazamiento lateral global de la estructura.

Si como criterio límite para evaluar el desempeño estructural se utiliza el de falla incipiente, el valor de $D M I_{P A}$ debe limitarse a ser menor que 1.0. A nivel global, la definición de falla adolece de las mismas inconsistencias descritas anteriormente para el caso de un elemento estructural. En este trabajo se considera que la falla incipiente global ocurre cuando el $D M I_{P A}$ promedio de todas las vigas del entrepiso mas dañado de la estructura alcanza un valor de 1.0. La falla incipiente a nivel global implica:

$$
\frac{\mu_{\max }}{\mu_{u}}+\beta \frac{N E_{H \mu}}{\mu_{u}}=1 \Rightarrow \mu_{\max }=\mu_{u}-\beta N E_{H \mu}
$$

La ec. 3 sugiere que el valor de $\mu_{\max }$ que puede desarrollar una estructura durante una excitación sísmica decrece conforme se incrementa la disipación de energía plástica (caracterizada por $N E_{H \mu}$ ). Este hecho tiene implicaciones importantes, porque plantea que el valor de $\mu_{\max }$ asociado al colapso o falla de la estructura puede variar y ser, en algunos casos, mucho menor que el valor de $\mu_{u}$. 
En el caso de estructuras con asimetría en fluencia, Terán et al. (1998, 2000) observan que el criterio utilizado para evaluar el desempeño sísmico de la estructura sismorresistente influye significativamente en la cuantificación de los factores de amplificación de resistencia. En particular, la importancia del daño producto de la disipación de energía en estructuras con asimetría en fluencia se reduce significativamente con respecto a aquel que produce en estructuras simétricas. Conforme se incrementa el nivel de asimetría, las demandas de $N E_{H \mu}$ pierden importancia dentro del contexto de la ec. 3 y, como consecuencia, el valor de $\mu_{\max }$ que una estructura con asimetría en fluencia puede acomodar durante la excitación sísmica se incrementa de manera importante conforme crece su nivel de asimetría. Como consecuencia, el incremento de resistencia requerido de acuerdo a la ec. 3 para mantener igual el nivel de daño en una estructura asimétrica respecto al estimado en una estructura simétrica, es mucho menor que el requerido para mantener sus demandas máximas de ductilidad iguales.

Para ilustrar lo anterior, la fig. 2 presenta espectros de resistencia (seudo-aceleración) para ductilidad máxima constante y comportamiento elasto-plástico perfecto. Dichos espectros corresponden al acelerograma registrado durante 1985 en la Secretaría de Comunicaciones y Transportes, dirección Este-Oeste (SCT EO) y a un porcentaje de amortiguamiento crítico, $\xi$, de 0.05. Se grafica el coeficiente sísmico contra $T / T_{g}$, donde $T$ es periodo de vibración y $T_{g}$ el periodo dominante de la excitación. La fig. 3 muestra espectros de resistencia para daño constante $\left(D M I_{P A}=1\right)$ para el mismo acelerograma, comportamiento histerético y porcentaje de amortiguamiento crítico. Para el caso de la fig. 3 , se consideró $\beta$ igual a 0.15 en la ec. 3 . Mientras los espectros de ductilidad y daño constante correspondientes a $\theta=0$ presentan diferencias importantes (las ordenadas para daño constante son mucho mayores alrededor de $T / T_{g}=1$ ) debido a la importancia del término $N E_{H \mu}$ en la ec. 2 para estructuras simétricas; los correspondientes a $\theta=0.10$ exhiben ordenadas similares, ya que las demandas de energía plástica tienden a perder importancia en sistemas con asimetría en fluencia importante. Note que el incremento de resistencia requerido para mantener la ductilidad máxima igual en las figs. $2 \mathrm{a}$ y $2 \mathrm{~b}$ es mucho mayor respecto al que se requiere para mantener el nivel de daño igual en las figs. $3 \mathrm{a}$ y $3 \mathrm{~b}$.
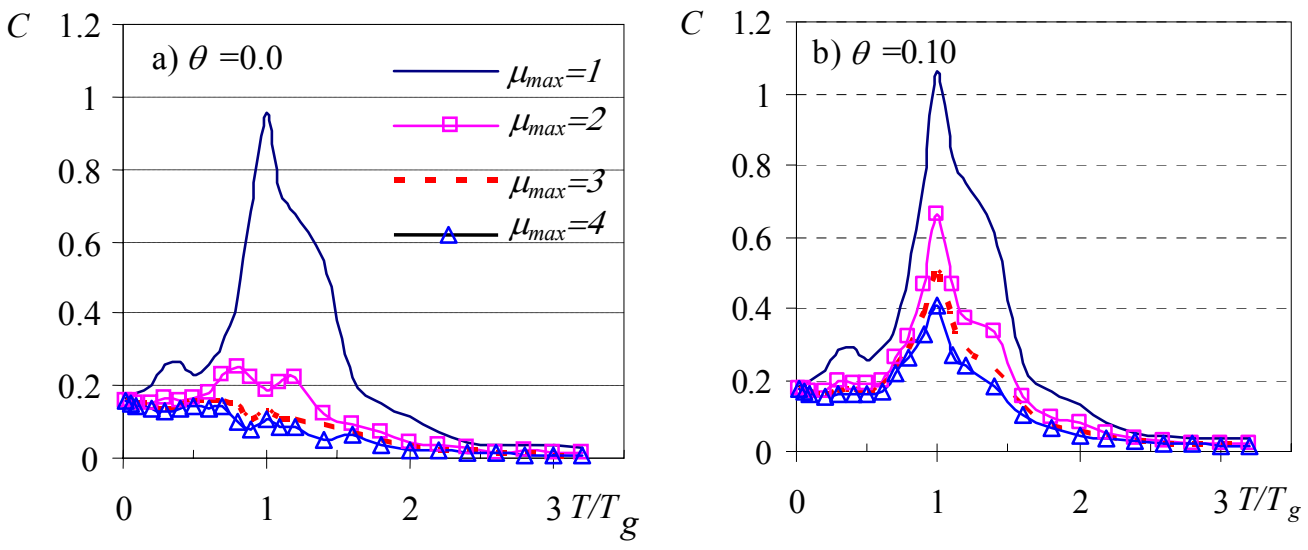

Figura 2. Efecto de la asimetría en fluencia en espectros de ductilidad constante, SCT EO, $\xi=0.05$

Cuando se considera un criterio de evaluación basado en la demanda máxima de deformación, se desprecia la diferencia que existe entre el comportamiento dinámico de sistemas 
simétricos y con asimetría en fluencia. Esto es, un criterio así no es capaz de balancear los efectos de un incremento importante en la demanda máxima de deformación con aquellos derivados de un decremento, igualmente importante, de las demandas plásticas acumuladas. Dada la importancia de la variación de las demandas acumuladas de deformación en sistemas asimétricos así como las tendencias de plantear métodos de diseño sísmico mas racionales, en este trabajo se evalúa el desempeño sísmico de los sistemas estructurales haciendo consideración explícita de las demandas de energía plástica (esto es, conforme a la ec. 3), y no conforme a un criterio de $\mu_{\max }$ constante.
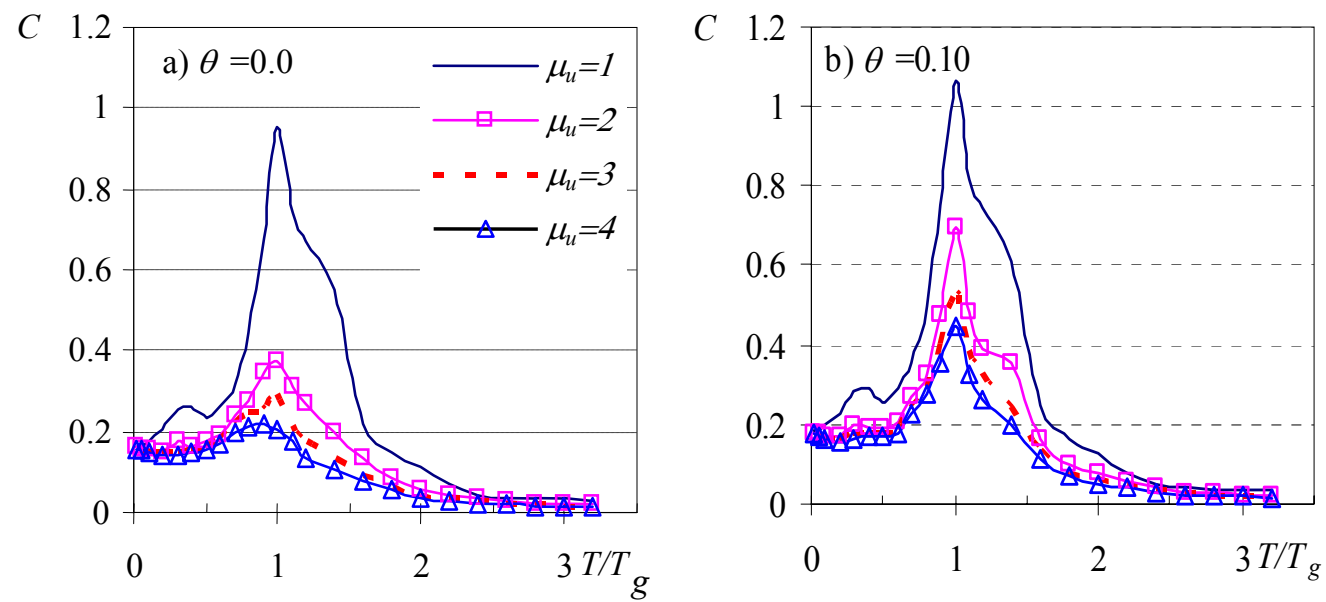

Figura 3. Efecto de la asimetría en fluencia en espectros de daño constante, SCT EO, $\xi=0.05$ $\beta=0.15$

\section{ESTUDIOS PREVIOS}

La respuesta dinámica de una estructura con asimetría en fluencia no puede entenderse a menos que se considere de manera sistemática las características de la estructura y de la excitación sísmica. En particular, la resistencia y comportamiento histerético de la estructura, el contenido de frecuencias y la duración de la excitación sísmica, y la interacción entre las características dinámicas de la estructura con aquellas de la excitación son relevantes para entender la respuesta dinámica de estructuras asimétricas (Ruiz et al. 1989, Terán et al. 1998, Terán-Gilmore et al. 2000).

En general, conforme menor sea la resistencia de una estructura, el efecto de la asimetría en fluencia será más significativo. Los sistemas con comportamiento elasto-plástico perfecto son particularmente susceptibles al efecto de la asimetría en fluencia, ya que tienden a exhibir un comportamiento que promueve que las demandas de deformación se acumulen en la misma dirección. La presencia de endurecimiento por deformación, fluencia gradual o degradación de rigidez se refleja en una reducción considerable del efecto de la asimetría en fluencia, ya que todos estos efectos promueven un comportamiento auto-centrado de la estructura que tiende a neutralizar el efecto de la asimetría en fluencia. 
El efecto de la asimetría en fluencia depende de manera importante de la duración de la excitación sísmica y del periodo del sistema, particularmente de la relación que $T$ guarda con el periodo dominante de la excitación. Al respecto, cuando la resistencia lateral de una estructura no se incrementa para contrarrestar el efecto de la asimetría en fluencia, se observan incrementos importantes en su demanda de ductilidad y en su nivel de daño, especialmente cuando su $T$ esta cercano a $T_{g}$. Conforme el valor de $T$ se aleja paulatinamente del valor de $T_{g}$, se aprecia una reducción en el efecto de la asimetría en fluencia. Para explicar esto, es necesario considerar que las demandas de energía, y por tanto las demandas acumuladas de deformación plástica tienden a ser mayores conforme $T$ se aproxima a $T_{g}$. Cuando los sistemas son simétricos tienden a oscilar alrededor de su posición no deformada y las demandas plásticas acumuladas tienden a contrarrestarse unas con otras. Sin embargo, los sistemas asimétricos, especialmente los que exhiben comportamiento elasto-plástico perfecto, tienden a acumular las demandas de deformación plástica en una sola dirección, de tal manera que las estructuras sujetas a altas demandas de energía tienden a ser mas susceptibles al efecto de la asimetría en fluencia. Esto también explica porque la asimetría en fluencia tiende a ser más importante en excitaciones de larga duración generadas en suelo blando, ya que su contenido de energía es mucho mayor que aquel correspondiente a las generadas en suelo firme.

\section{DEFINICIÓN}

Una estructura sismorresistente requiere diseñarse para una mayor resistencia lateral conforme crece su asimetría en fluencia (ver figs. 2 y 3). Por tanto, es conveniente definir un factor de amplificación de resistencia que permita el diseño de la resistencia lateral de este tipo de estructuras. Se define el factor de amplificación de resistencia $(A)$ para sistemas con asimetría en fluencia como:

$$
A\left(\mu_{0}, T_{0}, \theta_{0}\right)=\frac{C\left(\mu_{u}=\mu_{0}, T=T_{0}, \theta=\theta_{0}\right)}{C\left(\mu_{u}=\mu_{0}, T=T_{0}, \theta=0.0\right)}
$$

donde $\mu_{o}, \theta_{o}, T_{o}$ son los valores específicos de $\mu_{u}, \theta$ y $T$ asociados a la estructura para la cual se determina el valor de $A$, y $C$ el coeficiente sísmico de diseño correspondiente a un porcentaje de amortiguamiento crítico de 0.05 .

Si se conoce el valor de $A$, es posible estimar la resistencia que debe suministrarse a una estructura con asimetría en fluencia tomando como base el espectro inelástico para un sistema simétrico conforme a lo siguiente:

$C\left(\mu_{0}, T_{0}, \theta_{0}\right)=A\left(\mu_{0}, T_{0}, \theta_{0}\right) C\left(\mu_{o}, T_{0}, \theta=0\right)$

\section{NORMAS TÉCNICAS COMPLEMENTARIAS PARA DISEÑO POR SISMO}

Dada la problemática que la asimetría en fluencia representa para algunas estructuras ubicadas en la Ciudad de México, las NTCS del Reglamento de Construcciones del D.F. especifican en su 
Capítulo 11: "tratándose de estructuras cuyo comportamiento en sentidos opuestos sea asimétrico por inclinación de la estructura con respecto a la vertical, si el desplomo de la construcción excede de 0.01 veces su altura, se tomará en cuenta la asimetría multiplicando las fuerzas sísmicas de diseño por $1+5 Q f$ cuando se use el método de análisis estático o el dinámico modal"; donde $Q$ es el factor de comportamiento sísmico y $f$ el desplomo de la construcción dividido entre su altura (en este artículo $f$ se ha denotado $\alpha$ ).

En caso de que la asimetría en fluencia se deba a otra causa, las NTCS especifican en su Sección 8.8: "En el diseño de estructuras cuyas relaciones fuerza-deformación difieran en sentidos opuestos, se dividirán los factores de resistencia que corresponden según las normas respectivas, entre el siguiente valor: $1+2.5 d Q$, donde d es la diferencia en los valores de a/ $Q^{\prime}$, expresados como fracción de la gravedad, que causarían la falla o fluencia plástica de la estructura en uno y otro sentido de la dirección de análisis". Considerando que dentro del contexto de las NTCS, el valor de $d$ es equivalente a $f / 2$, puede concluirse que el nivel de diseño de una estructura asimétrica es consistente, independientemente de la causa de la asimetría.

Dado que dentro del contexto de las NTCS el valor de $Q$ se establece de manera independiente al periodo fundamental de vibración de la estructura, los factores $1+5 Q \alpha \mathrm{y}$ $1+2.5 d Q$ también son independientes del valor de $T$ para un nivel dado de asimetría. Además, los requerimientos enunciados arriba son válidos para las tres zonas consideradas en el Reglamento de Construcciones del D.F.: Zona de Lomas, Zona de Transición y Zona del Lago. Finalmente, las NTCS toman en cuenta la resistencia de la estructura con asimetría en fluencia a través del parámetro $Q$ (un mayor valor de $Q$ implica menor resistencia lateral de diseño). Por tanto, aunque las expresiones $1+5 Q \alpha$ y $1+2.5 d Q$ reflejan el hecho de que el efecto de la asimetría crece conforme se reduce la resistencia de la estructura, no hay una consideración explícita de variables, que como el tipo de suelo donde se construye la estructura, su comportamiento histerético y la relación que existe entre su periodo y el periodo dominante de la excitación, afectan significativamente la respuesta dinámica de estructuras con asimetría en fluencia.

Las expresiones actualmente incluidas en las NTCS dan lugar, en la mayoría de los casos, a diseños conservadores de estructuras con asimetría en fluencia. Terán-Gilmore et al. (2000) reportan que, con la excepción de estructuras que cumplen simultáneamente tres condiciones: A) Exhiben comportamiento similar al elasto-plástico perfecto, B) Su $T$ se aproxima al periodo de la excitación, y C) Se ven sujetas a excitaciones generadas en la Zona del Lago; el nivel de daño esperado en las estructuras diseñadas conforme a las NTCS decrece de manera importante conforme crece su nivel de asimetría. Si se considera que una estructura real difícilmente exhibirá un comportamiento similar al elasto-plástico perfecto, puede considerarse que los requerimientos de las NTCS para el diseño de estructuras con asimetría en fluencia suelen ser conservadores con respecto a los definidos para el diseño de estructuras simétricas en fluencia. Dado que el nivel de diseño de las NTCS para estructuras con asimetría en fluencia puede ir desde muy conservador hasta del lado de la inseguridad, es deseable hacer una revisión de los requerimientos de dichas normas. 


\section{ESTUDIOS EN SISTEMAS DE UN GRADO DE LIBERTAD}

Investigaciones recientes han mostrado que cuando la respuesta dinámica no lineal de marcos momento-resistentes esta dominada por su modo fundamental de vibración, es posible evaluar su respuesta dinámica y desempeño estructural global por medio de sistemas equivalentes de $1 \mathrm{GL}$ (Qi and Moehle 1991, Tso et al. 1993, Terán-Gilmore et al. 2000, Terán et al. 2002). En este sentido, la información aportada por el estudio de sistemas de $1 \mathrm{GL}$ es fundamental en el planteamiento de la resistencia lateral de diseño de estructuras complejas.

En esta sección se presentan expresiones para estimar el factor de amplificación de resistencia para estructuras con asimetría en fluencia ubicadas en la Ciudad de México. Estos factores se establecieron a partir de la respuesta de sistemas de $1 \mathrm{GL}$, la cual se estableció a su vez a partir del Método de Newmark con aceleración constante. Cabe mencionar que existen dos condiciones que deben considerarse para establecer la resistencia lateral de diseño de un sistema con asimetría en fluencia. Esto se debe a que el lado fuerte de la estructura puede estar orientado indistintamente en uno de dos sentidos; por ejemplo, considere que el desplomo de una estructura vista de frente puede orientarse hacia la izquierda o hacia la derecha. De antemano no es posible definir cual de estas dos condiciones resulta crítica para el desempeño de una estructura, por lo que cada sistema de $1 \mathrm{GL}$ considerado en este estudio se analizó dos veces, y la respuesta utilizada para determinar los factores de amplificación de resistencia corresponde a la condición crítica que surge de estos dos análisis. Continuando con el ejemplo, podría decirse que de antemano no es posible definir si un desplomo hacia la izquierda o hacia la derecha llevaría a la estructura a una condición más desfavorable, por lo que hay que analizar ambas condiciones, y sentar las bases de diseño sobre la condición más desfavorable.

Las expresiones que aquí se presentan se obtuvieron en dos etapas. Durante la primera se utilizaron acelerogramas sintéticos para estudiar el efecto que sobre el factor de amplificación $A$ tienen las distintas características mecánicas de la estructura sismorresistente, y la duración y contenido de frecuencias de la excitación sísmica. En la segunda fase, que consideró acelerogramas reales registrados en el Distrito Federal, se propusieron expresiones para estimar dicho factor. Los resultados obtenidos en la primera etapa ayudaron a determinar las variables que se incluyeron en el análisis de regresión realizado en la segunda etapa.

\section{Propiedades Estructurales}

En los estudios que se reportan, se consideraron sistemas de 1GL con comportamiento elastoplástico-perfecto y con comportamientos histeréticos que exhiben diferente nivel de degradación. La fig. 4, que muestra los cuatro comportamientos histeréticos con degradación considerados, deja ver que este trabajo no contempló adelgazamiento del ciclo histerético. De manera general, puede decirse que los modelos degradantes mostrados en la fig. 4 corresponden a elementos y estructuras de concreto reforzado con diferente detallado. Los ciclos histeréticos que se muestran fueron modelados a partir del modelo de los tres parámetros (Kunnath et al. 1990). Los valores de los tres parámetros asignados a cada uno de los modelos, y sus implicaciones, se resumen en la tabla 1. Vale la pena mencionar que el parámetro $\beta$ en la tabla, utilizado para degradar la resistencia del ciclo histerético, es el mismo considerado en el segundo término del índice de Park y Ang. Aunque 
el modelo de los tres parámetros original considera una envolvente tri-lineal que toma en cuenta explícitamente el agrietamiento del elemento de concreto reforzado, los resultados presentados en este artículo se obtuvieron a partir de una versión modificada que considera una envolvente bilineal. Lo anterior equivale a considerar que la estructura esta agrietada en el momento en que se le sujeta a la excitación sísmica, o dicho en otras palabras, que el periodo empleado durante los análisis corresponde a la rigidez asociada al punto de fluencia, y no a la rigidez inicial no agrietada de la estructura.

Se consideraron sistemas de $1 \mathrm{GL}$ con ductilidades últimas que oscilan entre 1 y 4 . La asimetría en fluencia se caracterizó por medio de valores de $\theta$ que oscilan entre 0.01 y 0.25 . Durante la formulación de expresiones para estimar $A$ en la Zona de Lomas se consideró un intervalo de $T$ de 0.1 a 5 segundos; mientras que en el caso de la Zona del Lago, se consideró un intervalo de $T / T_{g}$ de 0.1 a 5.0 .

a) Modelo $1 \quad F$

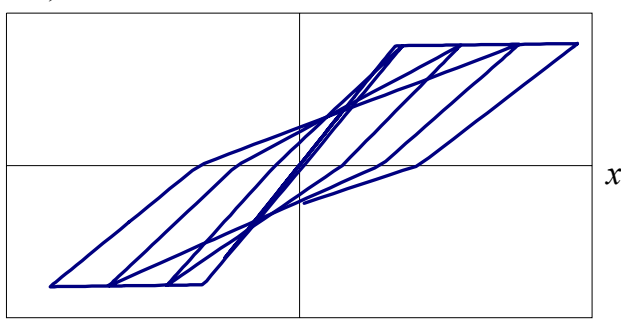

c) Modelo $3 \quad F$

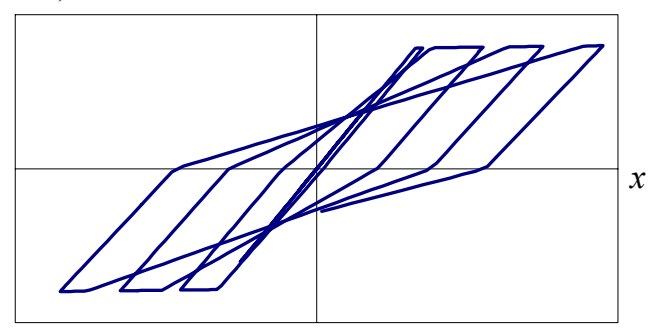

b) Modelo $2 \quad F$

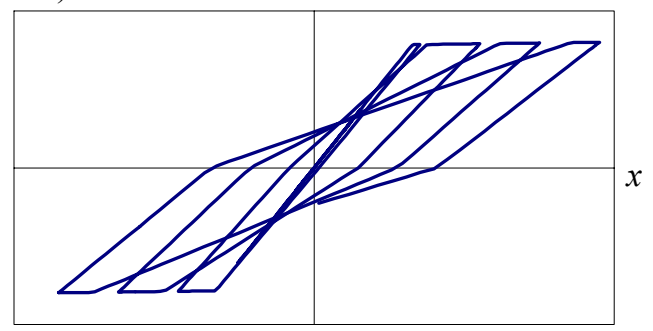

d) Modelo 4

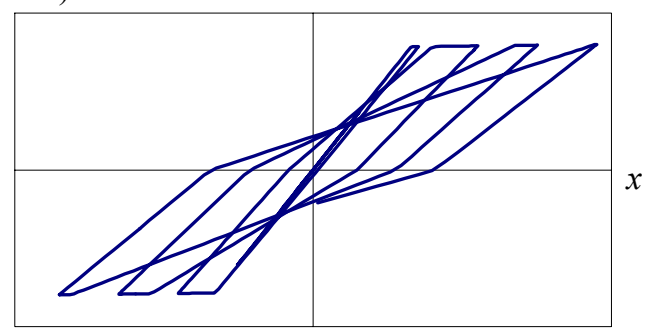

Figura 4. Comportamientos histeréticos degradantes considerados

Durante la definición de $A$ se contemplaron de manera implícita los efectos de segundo orden a través de considerar una pendiente post-elástica igual a cero. Para aclarar este punto, es necesario considerar que cuando se sujeta un edificio a un estado de deformación monotónicamente creciente, el endurecimiento por deformación de sus elementos estructurales, $\mathrm{y}$ el hecho de que no todos fluyen al mismo tiempo, se combinan para producir un pendiente postelástica positiva. Una vez que se toman en cuenta los efectos de segundo orden $(P-\Delta)$, los momentos secundarios que introducen las cargas gravitacionales tienden a contrarrestar los efectos del endurecimiento por deformación y de fluencia paulatina. En el caso de marcos momento-resistentes dúctiles diseñados conforme al Reglamento de Construcciones del D.F., la contraposición de los efectos de endurecimiento por deformación, fluencia paulatina y de segundo orden, resulta en una pendiente post-elástica prácticamente igual a cero en el intervalo de desplazamientos que va desde la fluencia global hasta el desplazamiento último de los marcos 
(Terán-Gilmore et al. 2000). Consistente con esto, en el estudio que se reporta se consideró una pendiente post-elástica de cero para los sistemas de $1 \mathrm{GL}$.

Tabla 1. Valor de los tres parámetros considerados para los diferentes modelos degradantes

\begin{tabular}{|c|c|c|c|c|c|c|}
\hline \multirow[b]{2}{*}{ Modelo } & \multicolumn{2}{|r|}{ Parámetro $\alpha$} & \multicolumn{2}{|r|}{ Parámetro $\beta$} & \multicolumn{2}{|r|}{ Parámetro $\gamma$} \\
\hline & Valor & Implicación & Valor & Implicación & Valor & Implicación \\
\hline 1 & 2 & $\begin{array}{l}\text { Degradación importante de } \\
\text { rigidez durante descarga }\end{array}$ & 0 & $\begin{array}{l}\text { No degradación de } \\
\text { resistencia }\end{array}$ & 1 & $\begin{array}{l}\text { No adelgazamiento } \\
\text { del ciclo histerético }\end{array}$ \\
\hline 2 & 2 & $\begin{array}{l}\text { Degradación importante de } \\
\text { rigidez durante descarga }\end{array}$ & 0.15 & $\begin{array}{l}\text { Estructura dúctil de } \\
\text { concreto reforzado }\end{array}$ & 1 & $\begin{array}{l}\text { No adelgazamiento } \\
\text { del ciclo histerético }\end{array}$ \\
\hline 3 & 10 & $\begin{array}{l}\text { Degradación pequeña de } \\
\text { rigidez durante descarga }\end{array}$ & 0.15 & $\begin{array}{l}\text { Estructura dúctil de } \\
\text { concreto reforzado }\end{array}$ & 1 & $\begin{array}{l}\text { No adelgazamiento } \\
\text { del ciclo histerético }\end{array}$ \\
\hline 4 & 2 & $\begin{array}{l}\text { Degradación importante de } \\
\text { rigidez durante descarga }\end{array}$ & 0.30 & $\begin{array}{l}\text { Estructura no- } \\
\text { dúctil de concreto } \\
\text { reforzado }\end{array}$ & 1 & $\begin{array}{l}\text { No adelgazamiento } \\
\text { del ciclo histerético }\end{array}$ \\
\hline
\end{tabular}

\section{Primera Etapa}

En esta etapa se definieron las características de las excitaciones sísmicas y las propiedades estructurales que deberían utilizarse durante las regresiones planteadas durante la segunda etapa. Además, la primera etapa sirvió para definir criterios de selección para los acelerogramas que conformaron las muestras utilizadas en dichas regresiones. Una discusión detallada de los resultados obtenidos en la primera etapa puede encontrarse en Arroyo y Terán (2001)

Se utilizaron 240 acelerogramas sintéticos, agrupados en cuatro grupos de 60 acelerogramas con duración de fase intensa de 10, 20, 30 y 50 segundos. Estos acelerogramas fueron generados filtrando ruidos blancos gaussianos a través de un filtro Kanai-Tajimi. Para cada duración, se generaron tres grupos de 20 acelerogramas, un grupo para la Zona de Lomas, otro para la Zona de Transición y finalmente un tercero para la Zona del Lago. Las frecuencias bajas de los acelerogramas se eliminaron por medio del filtro de Hodder. A los acelerogramas se les suministró una variación razonable en el tiempo de la intensidad sísmica al filtrarlos en el dominio del tiempo de acuerdo a las indicaciones de Tung et al. (1992). Una descripción más detallada de la generación de estos acelerogramas puede encontrarse en Terán-Gilmore (1996).

Los resultados más importantes derivados de la primera etapa pueden resumirse conforme a lo siguiente:

1) Los sistemas con simetría en fluencia presentan, particularmente en suelo blando, un comportamiento dinámico sustancialmente diferente al exhibido por sistemas con asimetría en fluencia. Mientras que las demandas acumuladas de deformación plástica suelen ser de importancia en sistemas simétricos, los sistemas asimétricos exhiben una historia de fuerza-deformación que se asemeja mucho un estado de deformación monotónicamente creciente. Por tanto, el planteamiento de expresiones para estimar $A$ requiere contemplar un criterio de evaluación estructural que considere explícitamente las demandas acumuladas de deformación plástica. 
2) Existe una clara dependencia de los valores de $A$ con respecto al contenido de frecuencias de la excitación sísmica. La segunda etapa requiere establecer muestras de acelerogramas reales para diferentes tipos de suelo. Cuando se utiliza un criterio de daño constante, la duración de fase intensa de la excitación no tiene una influencia importante en $A$. La conformación de las muestras de acelerogramas reales con fines de regresión puede llevarse a cabo sin consideración explícita de la duración de los acelerogramas.

3) El valor de $A$ depende de las características dinámicas de la estructura y de su interacción con las características dinámicas de la excitación sísmica. Una expresión para estimar $A$ debe hacer consideración explícita del periodo de la estructura.

4) Cuando se utiliza un criterio de daño constante, un decremento en la resistencia lateral de la estructura se refleja en un ligero incremento de su valor de $A$, particularmente para $T$ cercano a $T_{g}$. La dependencia del valor de $A$ con respecto a la resistencia del oscilador es relativamente débil, de tal manera que es posible estimarlo razonablemente sin consideración explícita de la resistencia lateral de la estructura. Dado que la resistencia de la estructura depende de $\mu_{\max }$ ó $\mu_{u}$ (según el criterio de evaluación estructural utilizado), es posible decir que puede estimarse razonablemente bien el valor de $A$ sin consideración explícita de la ductilidad de la estructura.

5) Los valores de $A$ correspondientes a comportamiento elasto-plástico perfecto son mucho mayores que los observados en sistemas degradantes. Mientras no haya adelgazamiento del ciclo histerético, los valores de $A$ obtenidos para sistemas degradantes son sensiblemente independientes de la velocidad con que se degrada su rigidez y resistencia. La estimación razonable del valor de $A$ requiere plantear por lo menos dos series de ecuaciones: una aplicable a sistemas con comportamiento elasto-plástico perfecto, y otro aplicable a sistemas con degradación del ciclo histerético.

\section{Segunda Etapa}

En la segunda etapa se plantearon expresiones para estimar el valor de $A$ en función de los parámetros identificados como relevantes en la primera etapa. En particular, el contenido de frecuencias de la excitación se consideró a través de plantear expresiones separadas para las Zonas de Lomas y del Lago del D.F. Aunque en este artículo no se incluyen, es posible plantear expresiones similares para la Zona de Transición. En cuanto a las propiedades estructurales, se consideraron explícitamente el periodo de la estructura y su nivel de asimetría en fluencia. El comportamiento histerético de la estructura se contempla al plantear regresiones separadas para sistemas con comportamiento elasto-plástico perfecto y para sistemas con degradación del ciclo histerético. Los valores de $A$ considerados con fines de regresión se establecieron a partir de un criterio de daño constante. En particular, se utilizó la ec. 3 con $\beta=0.15$.

Las expresiones se plantearon a partir de un análisis de regresión de tal manera que se minimizó en lo posible el error cuadrático medio. Cabe mencionar que las expresiones resumidas en este trabajo se derivaron al modificar ligeramente (para hacer más sencilla su aplicación práctica) las expresiones obtenidas directamente del análisis de regresión. 
Para establecer la expresión correspondiente a la Zona de Lomas, se utilizaron 20 acelerogramas registrados en diferentes sitios de dicha zona. La muestra incluyó acelerogramas con aceleraciones máximas entre $0.01 \mathrm{~g}$ y $0.04 \mathrm{~g}$. En cuanto a la expresión aplicable a la Zona del Lago, se utilizaron 25 acelerogramas registrados en diferentes sitios ubicados en dicha zona. Para cada acelerograma, $T_{g}$ se definió como el valor de $T$ en que su espectro elástico de energía de entrada para $\xi$ de 0.05 alcanza su máximo. La muestra incluyó acelerogramas con aceleraciones máximas entre $0.03 \mathrm{~g}$ y $0.16 \mathrm{~g}$, y con $T_{g}$ entre 1.9 y 7 segundos.

Arroyo y Terán (2001) presentan información de todos los acelerogramas reales utilizados en el estudio que aquí se reporta, y discuten de manera general sus características dinámicas. La fig. 5 presenta, para $\xi$ de 0.05 , la media y coeficiente de variación (COV) de los espectros elásticos de resistencia normalizados por la aceleración máxima del terreno. Las ordenadas de un espectro normalizado se denotan $c_{\text {norm }}$, y se obtienen al normalizar las ordenadas de un espectro de resistencia por el valor de su ordenada al origen (esto implica que la ordenada al origen de un espectro normalizado es igual a uno). La figura incluye resultados para las muestras correspondientes a las Zonas de Lomas y del Lago. Para el caso de la Zona de Lomas, fig. 5a, se observa una amplificación máxima de la aceleración del terreno cercana a 2.5 para un periodo dominante cercano a $0.8 \mathrm{seg}$. Las tendencias mostradas no corresponden del todo a excitaciones "típicas" generadas en suelo firme, particularmente porque los espectros normalizados de estas suelen alcanzar su ordenada máxima para periodos alrededor de $0.4 \mathrm{seg}$. Además, la fig. 5a presenta ordenadas mucho mayores, en un intervalo de $T$ que abarca de 2 a 4 segundos, que aquellas exhibidas por excitaciones "típicas" de suelo firme. Puede concluirse que el espectro mostrado en la fig. 5a presenta particularidades muy notorias debido a las condiciones que se presentan en la Zona de Lomas del D.F., en donde los movimientos del terreno generados en suelo firme presentan un contenido importante de ondas de baja frecuencia.

Para la muestra de la Zona del Lago, la fig. 5b muestra una amplificación máxima de la aceleración del terreno cercana a 4.5 para $T / T_{g}$ cercano a 1.0. El espectro promedio para la Zona del Lago incluye un segundo máximo en $T / T_{g}$ cercano a 0.3 , ya que muchos acelerogramas en la muestra exhibieron más de un pico en su espectro de resistencia (fenómeno posiblemente relacionado con un segundo modo de vibración del suelo blando). Las figs. 5c y 5d muestran los COV asociados a los espectros mostrados en las figs. 5 a y $5 \mathrm{~b}$, respectivamente. Para el intervalo de periodos considerado, se observa un COV relativamente bajo para las excitaciones correspondientes a la Zona de Lomas. En el caso de las excitaciones registradas en la Zona del Lago, se observan valores altos de COV para valores de $T / T_{g}$ mayores que dos.

Una vez conformadas las muestras de acelerogramas reales, se realizaron una serie de análisis de regresión para proponer expresiones que, en función de las variables identificadas como importantes durante la primera etapa del estudio, sean capaces de estimar razonablemente el valor de $A$. Despues de algunas propuestas preliminares, se consideró estimar el valor de $A$ en la Zona del Lago a partir de la siguiente expresión: 
$A=\frac{b\left(T / T_{g}\right)^{a}}{c+\left|\frac{T}{T_{g}}-1\right|}+d$
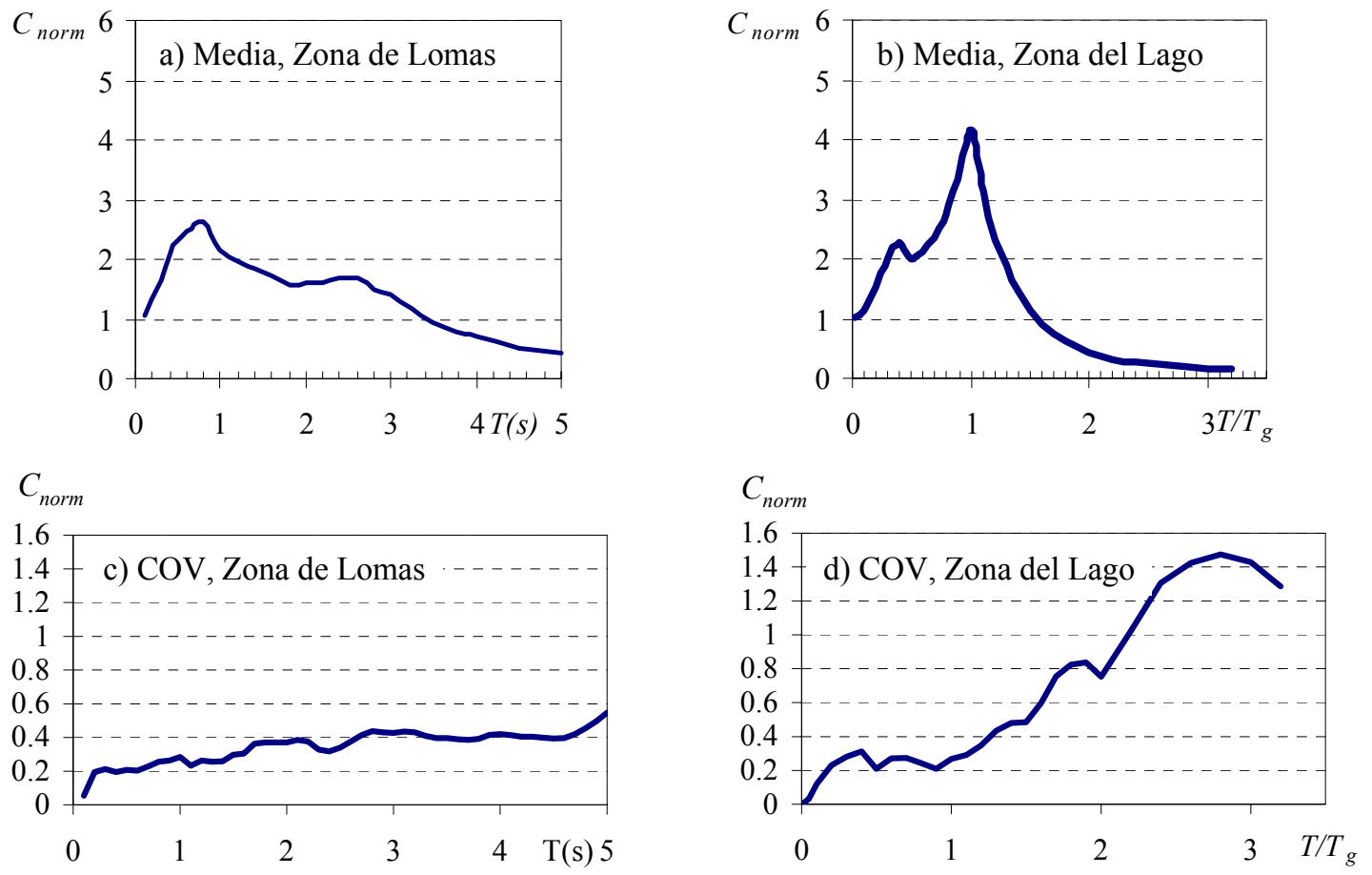

Figura 5. Espectros elásticos de resistencia normalizados, acelerogramas reales, $\xi=0.05$

La forma básica $\left(T / T_{g}\right)^{a} /\left(c+/ T / T_{g}-1 /\right)$ tiende a cero para $T$ de cero y adquiere valores altos cuando $T / T_{g}$ se aproxima al valor de uno. El valor de $d$ representa entonces el valor de la ordenada al origen de un espectro de $A$. El valor del máximo que alcanza la curva depende de los valores de $a, b$ y $c$, mientras que el valor del exponente $a$ define la velocidad de caída del valor de $A$ a partir de ese máximo. Mediante un análisis de regresión estadística para minimizar el error cuadrático, se obtuvieron los valores para $a, b, c$ y $d$ resumidos en las dos primeras columnas de la tabla 2. El letrero EPP se refiere a comportamiento elasto-plástico perfecto, mientras que los valores indicados para comportamiento degradante son aplicables a sistemas con diferente nivel de degradación de rigidez y resistencia.

Tabla 2. Valor de parámetros involucrados en las ecs. 6 y 7

\begin{tabular}{|c|c|c|c|c|}
\hline \multirow{2}{*}{ Parámetro } & \multicolumn{2}{|c|}{ Zona del Lago } & \multicolumn{2}{c|}{ Zona de Lomas } \\
\cline { 2 - 5 } & EPP & Degradante & EPP & Degradante \\
\hline$a$ & 0.6 & 1.5 & 0.6 & 1.5 \\
\hline$b$ & $\theta(1.7-2.9 \theta)$ & $\theta(4.2-8.6 \theta)$ & $\theta(0.25-0.1 \theta)$ & $\theta$ \\
\hline$c$ & 0.2 & 0.6 & 0.2 & 0.6 \\
\hline$d$ & $1+\theta$ & $1+\theta$ & $1+\theta$ & $1+\theta$ \\
\hline
\end{tabular}


La fig. 6 compara, para comportamiento elasto-plástico perfecto y la muestra correspondiente a la Zona del Lago, valores medios de $A$ con aquellos arrojados por la ec. 6 . Aunque se observa una buena coincidencia, la ec. 6 tiende a sobreestimar ligeramente el valor de $A$ para combinaciones de valores grandes de $\theta$ y valores pequeños de $\mu_{u}$.

Cabe mencionar que la ec. 6 pudiera hacerse más compleja de manera que tome en cuenta un mayor número de parámetros y, por tanto, refleje mejor las tendencias observadas en la fig. 6 . Por ejemplo, dicha figura muestra que conforme el valor de $\theta$ se incrementa, las curvas de $A$ correspondiente a diferentes valores de $\mu_{u}$ tienden a separarse, de tal manera que podría considerarse conveniente plantear una expresión que resulte en diferentes valores de $A$ en función del valor de $\mu_{u}$. Al respecto, los autores consideran que la ec. 6 es suficientemente sencilla para una aplicación práctica, y que arroja resultados suficientemente precisos dentro del contexto de un diseño sísmico práctico.

La fig. 6 presenta además los valores de $A$ derivados de las NTCS para marcos dúctiles, diseñados para la Zona del Lago, y que exhiben asimetría en fluencia debido a un desplomo significativo. Para obtener estos valores, se ha supuesto que el coeficiente sísmico de diseño para estos marcos es igual a $c=\frac{a}{Q}=\frac{0.4}{4}=0.1$, y que la sobrerresistencia lateral esperada en los marcos dúctiles es de 100\% (Terán 1998). De esta manera, la resistencia lateral esperada en la estructura es el doble de la resistencia lateral de diseño, lo que resulta en un coeficiente sísmico de 0.2. El grado de asimetría en fluencia en estos marcos se caracteriza mediante la variable $\theta=\frac{\alpha}{c}=\frac{\alpha}{0.2}$, donde $\alpha$ es el desplomo de la construcción dividido entre su altura. La tabla 3 muestra los valores de $A$ derivados, bajo estas circunstancias, a partir de las NTCS.

La fig. 6 confirma que las expresiones actualmente incluidas en las NTCS suelen dar lugar a diseños conservadores de estructuras con asimetría en fluencia, y que una excepción a esto se da en estructuras que cumplen simultáneamente tres condiciones: A) Exhiben comportamiento similar al elasto-plástico-perfecto, B) Su $T$ se aproxima al periodo de la excitación $\left(T / T_{g} \approx 1\right), \mathrm{y}$ C) Se ven sujetas a excitaciones generadas en la Zona del Lago. Note que el nivel de conservadurismo de las expresiones incluidas en las NTCS se incrementa conforme crece el nivel de asimetría en fluencia. Vale la pena resaltar que en la fig. 6 se han comparado propuestas que han sido derivadas de criterios diferentes: mientras que los valores medios de $A$ y aquellos obtenidos con la ec. 6 han sido derivados de un criterio de daño constante; las expresiones incluidas en las NTCS están basadas en una combinación de resultados derivados de un criterio de ductilidad constante y juicio ingenieril. Una vez que los requerimientos de las NTCS se enmarcan dentro de criterios modernos de evaluación del desempeño estructural, es posible establecer la pertinencia de actualizarlos.

La fig. 7 muestra los coeficientes de variación (COV) de los valores de $A$ derivados del Modelo elásto-plástico perfecto. Estos valores, sugieren que la variabilidad de $A$ es muy pequeña, y mucho menor que la implicada por los valores de COV mostrados en la fig. $5 \mathrm{~d}$. 

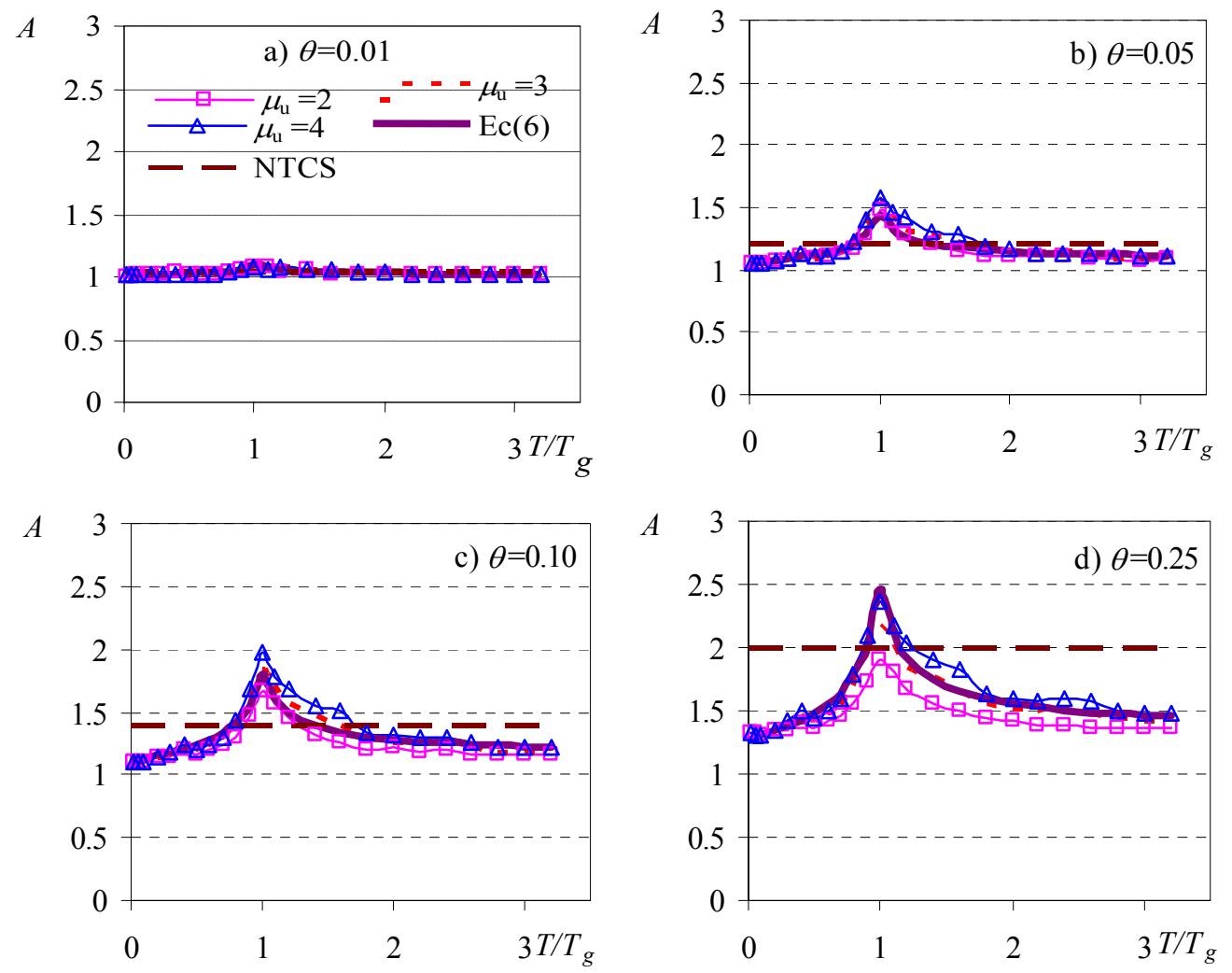

Figura 6. Comparación entre valores medios de $A$ y los valores obtenidos con la ec. 6, Zona del Lago, comportamiento elasto-plástico perfecto

Tabla 3. Valores de $A$ para marcos dúctiles, Zona del Lago

\begin{tabular}{|c|c|c|}
\hline$\theta$ & $\alpha=0.2 \theta$ & $A=1+5 Q \alpha$ \\
\hline 0.01 & 0.002 & 1.04 \\
\hline 0.02 & 0.010 & 1.20 \\
\hline 0.10 & 0.020 & 1.40 \\
\hline 0.25 & 0.050 & 2.00 \\
\hline
\end{tabular}

Las figs. 8 a 11 comparan, para comportamiento histerético degradante y la muestra correspondiente a la Zona del Lago, valores medios de $A$ con aquellos arrojados por la ec. 6 . Estas figuras incluyen resultados derivados a partir de los cuatro modelos histeréticos ilustrados en la fig. 4. Note que una vez mas, el valor de $A$ tiende a ser independiente de $\mu_{u}$ y tiende a crecer conforme el valor de $T / T_{g}$ se acerca a uno. Al comparar los resultados presentados en las figs. 8 a 11, puede concluirse que las particularidades del comportamiento histerético no afectan de manera importante al valor de $A$. Se observa además una buena coincidencia entre los valores de $A$ arrojados por la ec. 6 y los valores medios de $A$.

Las figs. 8 a 11 presentan además los valores de $A$ derivados de las NTCS para marcos dúctiles, diseñados para la Zona del Lago, y que exhiben asimetría en fluencia debido a un desplomo significativo. Note que estos valores corresponden a los resumidos en la tabla 3, ya que las NTCS no consideran el comportamiento histerético de la estructura en el cálculo de $A$. Puede 
apreciarse la naturaleza conservadora de las expresiones incluidas en las NTCS, particularmente para estructuras con $T$ alejado de $T_{g}$ y valores grandes de $\theta$.
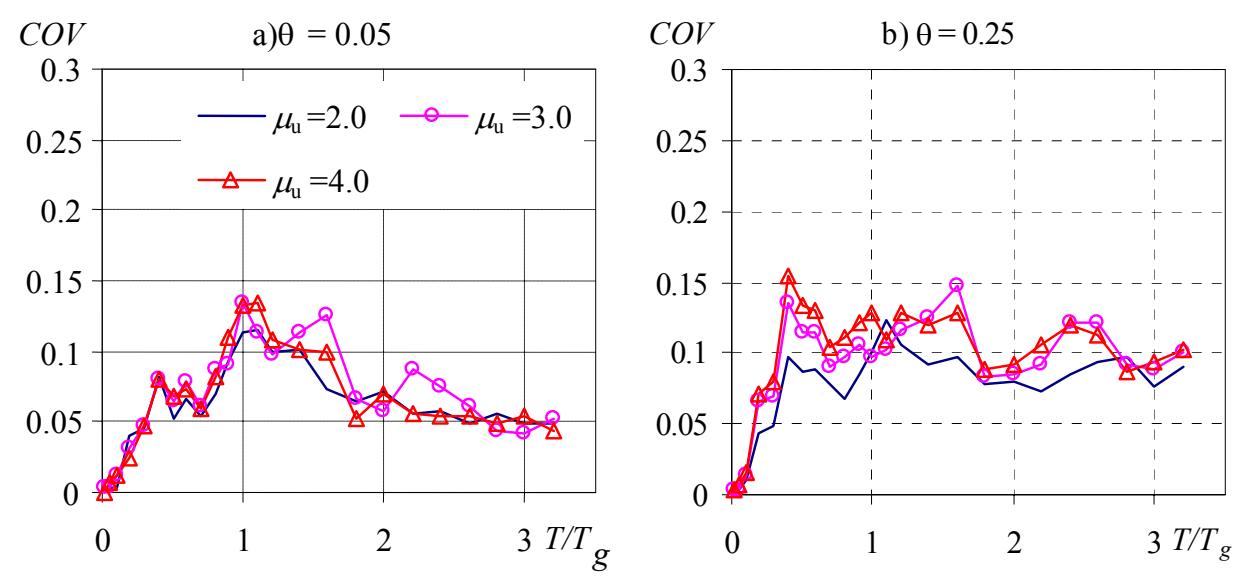

Figura 7. COV de A, Zona de Lago, Caso elásto-plástico perfecto
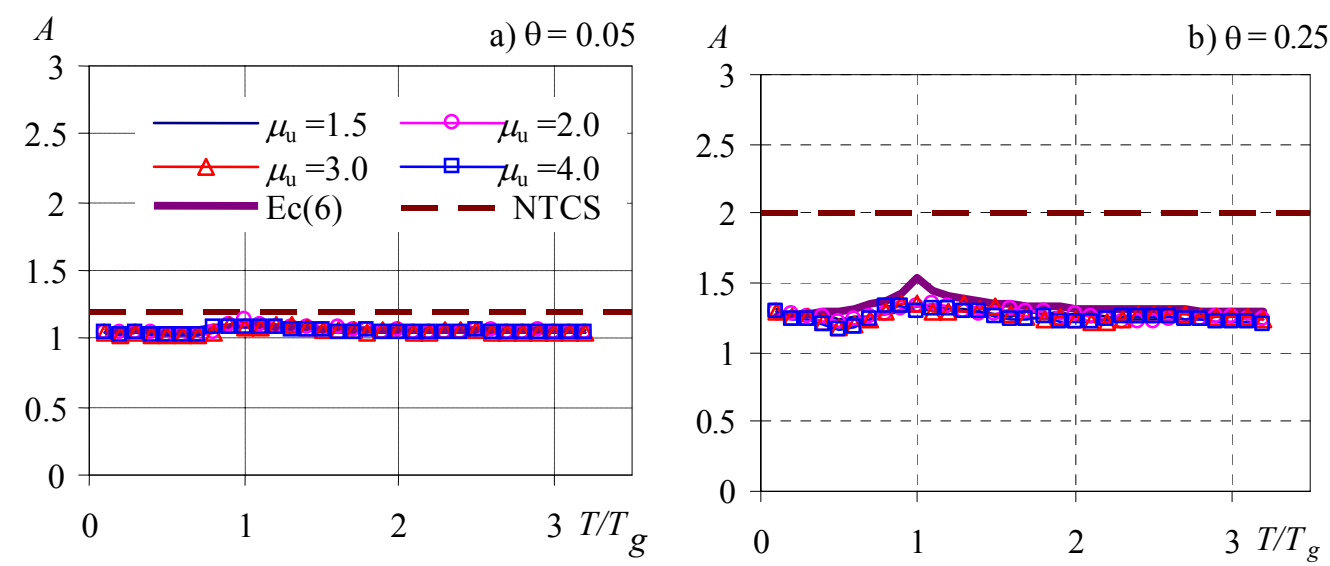

Figura 8. Comparación entre valores medios de $A$ y los valores obtenidos con la ec. 6, Zona del Lago, Modelo 1
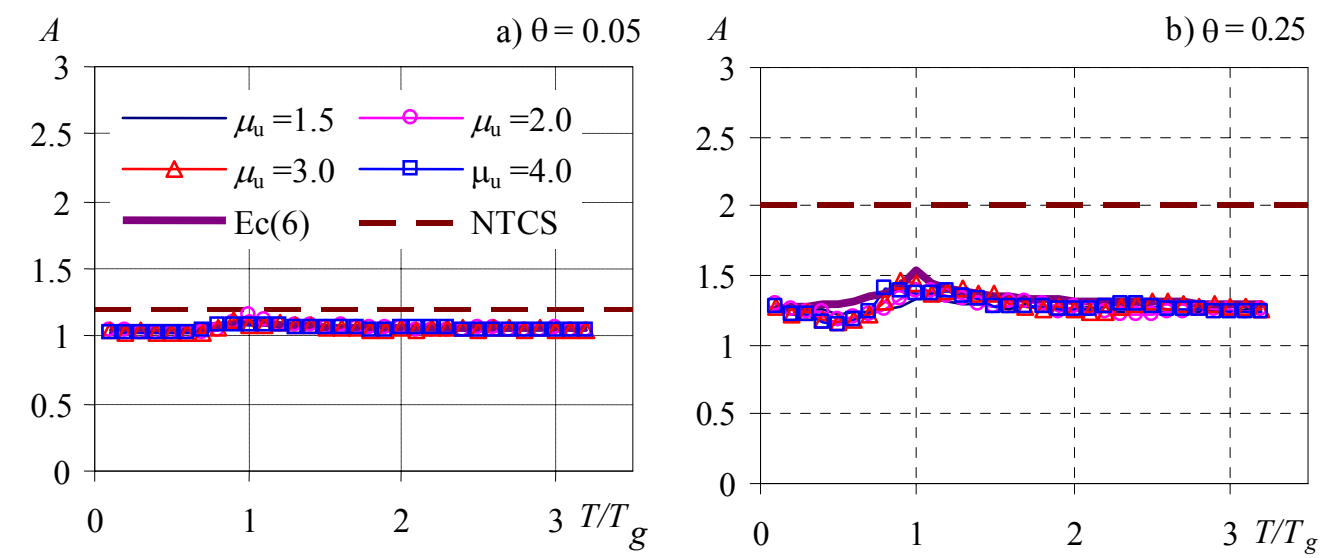

Figura 9. Comparación entre valores medios de $A$ y los valores obtenidos con la ec. 6, Zona del Lago, Modelo 2 

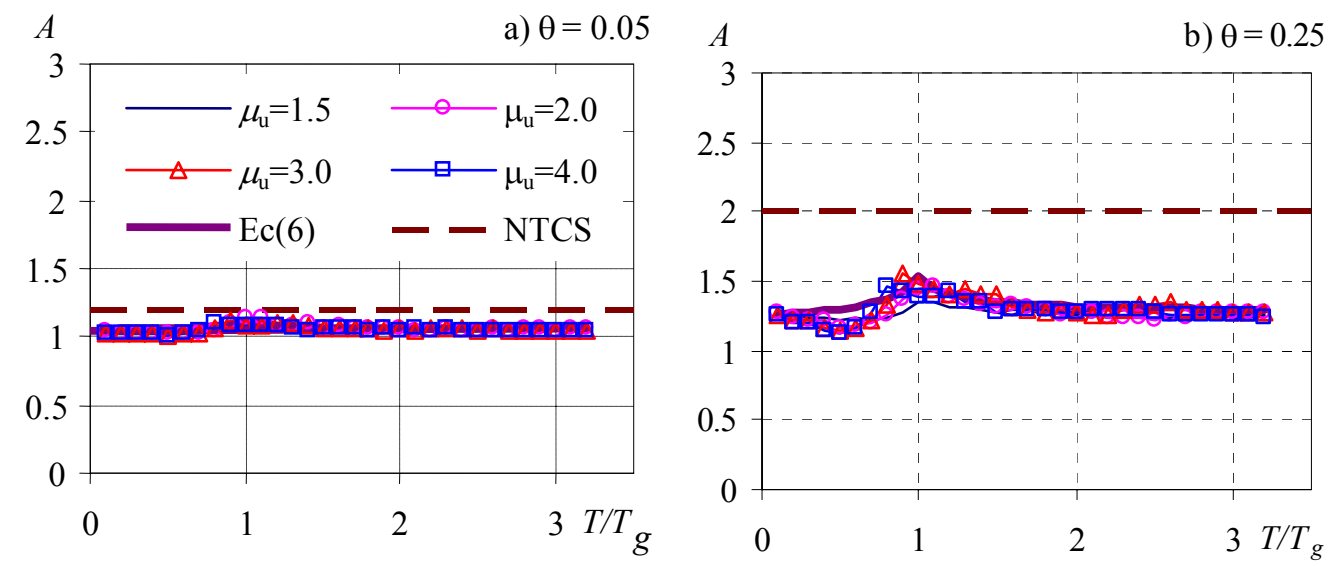

Figura 10. Comparación entre valores medios de $A$ y los valores obtenidos con la ec. 6, Zona del Lago, Modelo 3
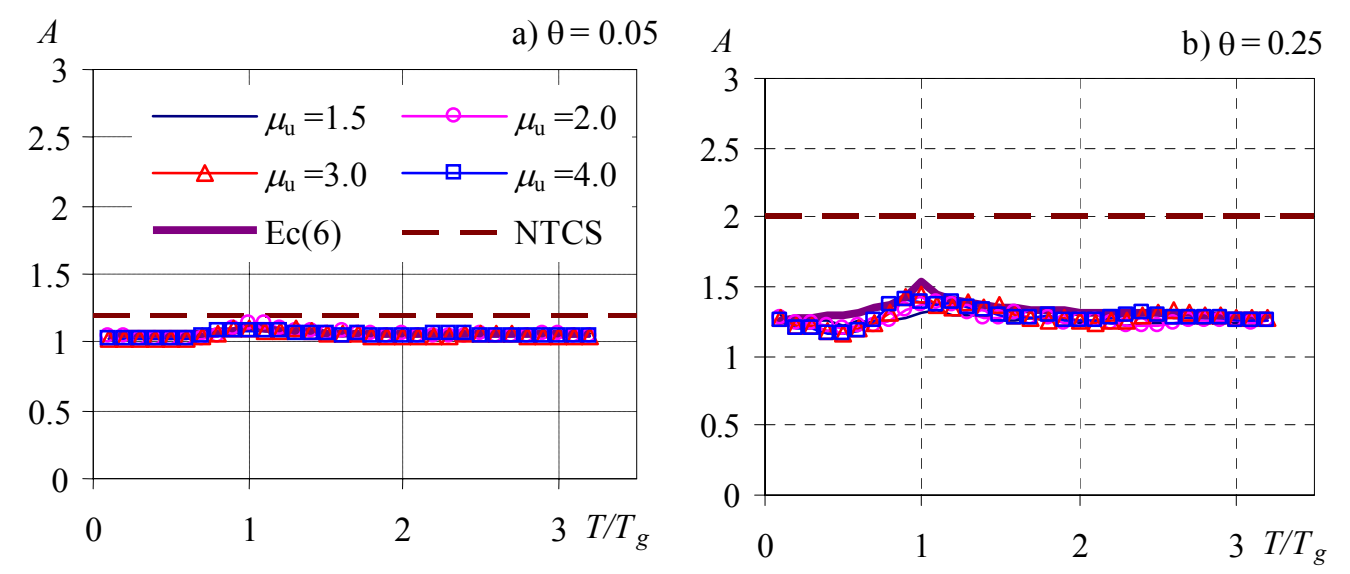

Figura 11. Comparación entre valores medios de $A$ y los valores obtenidos con la ec. 6, Zona del Lago, Modelo 4
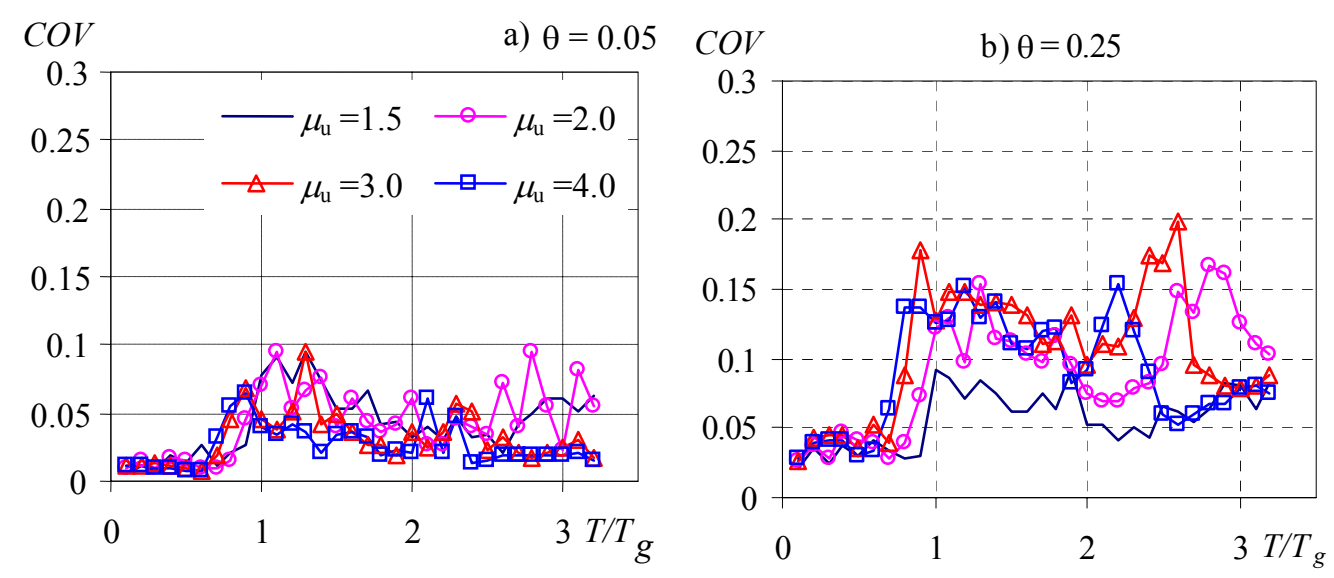

Figura 12. COV de A, Zona del Lago, Modelo 3 
La fig. 12 resume los coeficientes de variación (COV) de los valores de $A$ derivados del Modelo 3. Estos valores, que son similares a los obtenidos a partir de los otros modelos histeréticos degradantes utilizados, sugieren una vez más que la variabilidad de $A$ es muy pequeña.

En cuanto a la Zona de Lomas, se consideró estimar el valor de $A$ a partir de una familia de curvas con la siguiente forma:

$$
A=\frac{b T^{a}}{c+T^{a}}+d
$$

La forma básica $\left(b T^{a}\right) /\left(c+T^{a}\right)$ tiende a cero para $T$ de cero y tiende al valor de $b$ conforme crece el valor de $T$. Entonces $d$ representa el valor de la ordenada al origen. El valor del máximo que alcanza la curva depende de los valores de $b$ y $d$, mientras que los valores de $a$ y $c$ definen la rapidez con que $A$ crece a partir de $T$ de cero. Mediante un análisis de regresión estadística se obtuvieron los valores para $a, b, c$ y $d$ resumidos en las dos segundas columnas de la tabla 2. Cabe mencionar que en el caso de sistemas con degradación de rigidez y resistencia los parámetros mostrados en la tabla 2 no corresponden al error cuadrático mínimo. Aunque la función $A=1+\theta$ resulta en errores cuadráticos sustancialmente menores, los autores consideran apropiado conservar para sistemas degradantes el formato usado para sistemas elasto-plásticos con el fin de no complicar demasiado la aplicación práctica de las expresiones propuestas.

La fig. 13 compara, para comportamiento elasto-plástico perfecto y la muestra correspondiente a la Zona de Lomas, valores medios de $A$ con aquellos arrojados por la ec. 7 . Aunque se observa una buena coincidencia, la ec. 7 tiende a sobreestimar el valor de $A$ para valores grandes de $\theta$ combinados con valores pequeños de $\mu_{u}$; y a subestimarlo ligeramente en el caso de estructuras con $T$ cercano a $3 \mathrm{seg}$, y valores grandes de $\theta$ y $\mu_{u}$. Como se comentó en el caso de la ec. 6, la ec. 7 puede refinarse para tomar en cuenta un mayor número de parámetros $\mathrm{y}$, por tanto, para reflejar mejor las tendencias observadas en la fig. 13.

La fig. 13 presenta además los valores de $A$ derivados de las NTCS para marcos dúctiles, diseñados para la Zona de Lomas. Se ha supuesto que el coeficiente sísmico de diseño para estos marcos es igual a $c=\frac{a}{Q}=\frac{0.16}{4}=0.04$. Aunque no se encontraron estadísticas al respecto, la información disponible a los autores indica que la sobrerresistencia de marcos dúctiles diseñados en la Zona de Lomas es mayor a la que corresponde a marcos dúctiles diseñados en la Zona del Lago (Gutiérrez 2002). Considerando una sobrerresistencia de 150\% para marcos dúctiles diseñados en la Zona de Lomas, la resistencia lateral esperada es de 2.5 la resistencia lateral de diseño, lo que resulta en un coeficiente sísmico $c$ de 0.10 . El grado de asimetría en fluencia en estos marcos se caracteriza mediante la variable $\theta=\frac{\alpha}{c}=\frac{\alpha}{0.10}$. La tabla 4 muestra los valores de $A$ derivados, bajo estas circunstancias, a partir de las NTCS. 

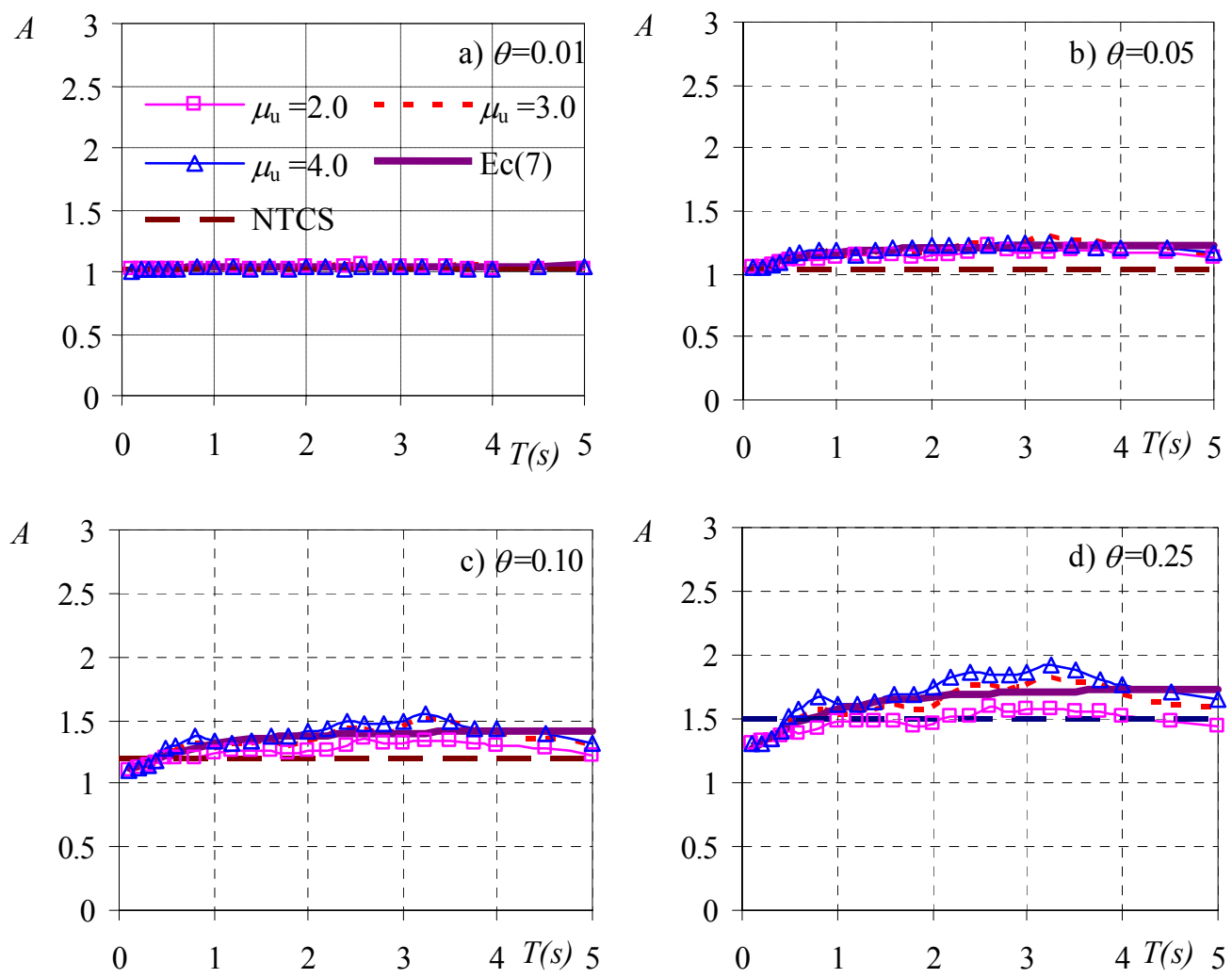

Figura 13. Comparación entre valores medios de $A$ y los valores obtenidos con la ec. 7, Zona de Lomas, comportamiento elasto-plástico perfecto

Tabla 4. Valores de $A$ para marcos dúctiles, Zona de Lomas

\begin{tabular}{|c|c|c|}
\hline$\theta$ & $\alpha=0.10 \theta$ & $A=1+5 Q \alpha$ \\
\hline 0.01 & 0.001 & 1.02 \\
\hline 0.02 & 0.002 & 1.04 \\
\hline 0.10 & 0.010 & 1.20 \\
\hline 0.25 & 0.025 & 1.50 \\
\hline
\end{tabular}

A partir de las comparaciones de las tablas 3 y 4, puede concluirse que el incremento de resistencia requerido para marcos dúctiles ubicados en la Zona de Lomas es aproximadamente la mitad del requerido para dichos marcos en la Zona del Lago. Conforme se podrá apreciar en ambas tablas, esto se debe a que el menor coeficiente sísmico de diseño para los marcos en la Zona de Lomas implica que alcanzan los valores de $\theta$ considerados en la tabla para valores menores de $\alpha$.

A diferencia de lo observado por Terán et al. (1998, 2000), la fig. 13 muestra que las expresiones actualmente incluidas en las NTCS dan lugar, para comportamiento elasto-plástico perfecto, a diseños del lado de la inseguridad para estructuras con asimetría en fluencia desplantadas en suelo firme. El problema, no detectado con anterioridad, surge a partir de que los requerimientos de las NTCS no contemplan la diferencia que existe entre el coeficiente sísmico de diseño para las diferentes zonas del D.F.; esto es, dichos requerimientos caracterizan el nivel 
de asimetría en fluencia en estructuras que exhiben desplomo mediante el valor de $\alpha$, no mediante el valor de $\theta$.

La fig. 14 muestra los coeficientes de variación de los valores de $A$ derivados del Modelo 3. Estos valores confirman la baja variabilidad de los valores de $A$, particularmente si se les compara con los valores de COV resumidos en la fig. 5c.
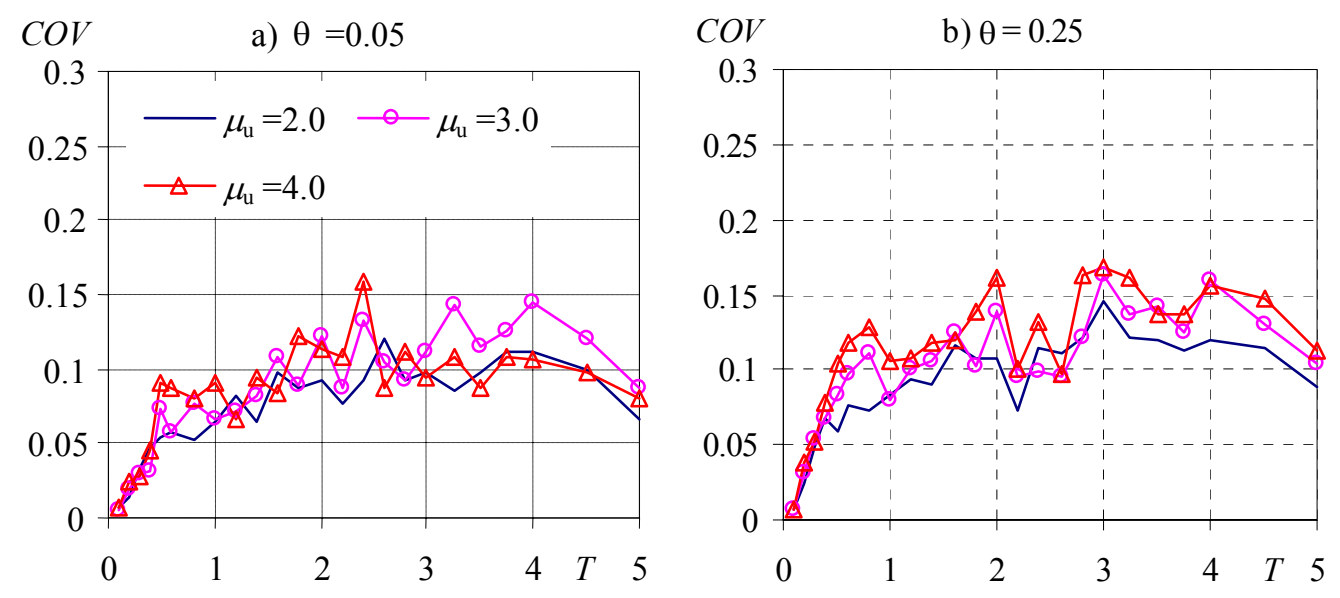

Figura 14. COV de A, Zona de Lomas, Caso elásto-plástico perfecto

Las figs. 15 a 18 comparan, para comportamiento histerético degradante y la muestra correspondiente a la Zona de Lomas, valores medios de $A$ con aquellos arrojados por la ec. 7 . Estas figuras incluyen resultados derivados a partir de los cuatro modelos histeréticos ilustrados en la fig. 4. Note una vez más que el valor de $A$ tiende a ser independiente de $\mu_{u} \mathrm{y}$ de las particularidades del comportamiento histerético. Se observa una buena coincidencia entre los valores de $A$ arrojados por la ec. 7 y los valores medios de $A$.
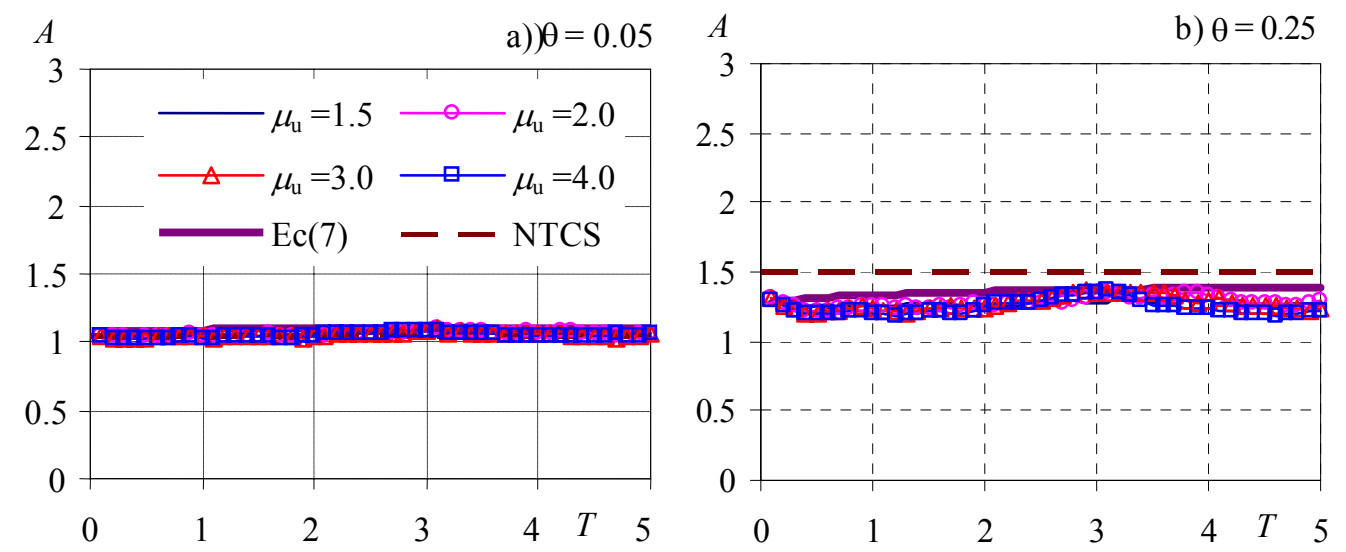

Figura 15. Comparación entre valores medios de $A$ y los valores obtenidos con la ec. 7, Zona de Lomas, Modelo 1 

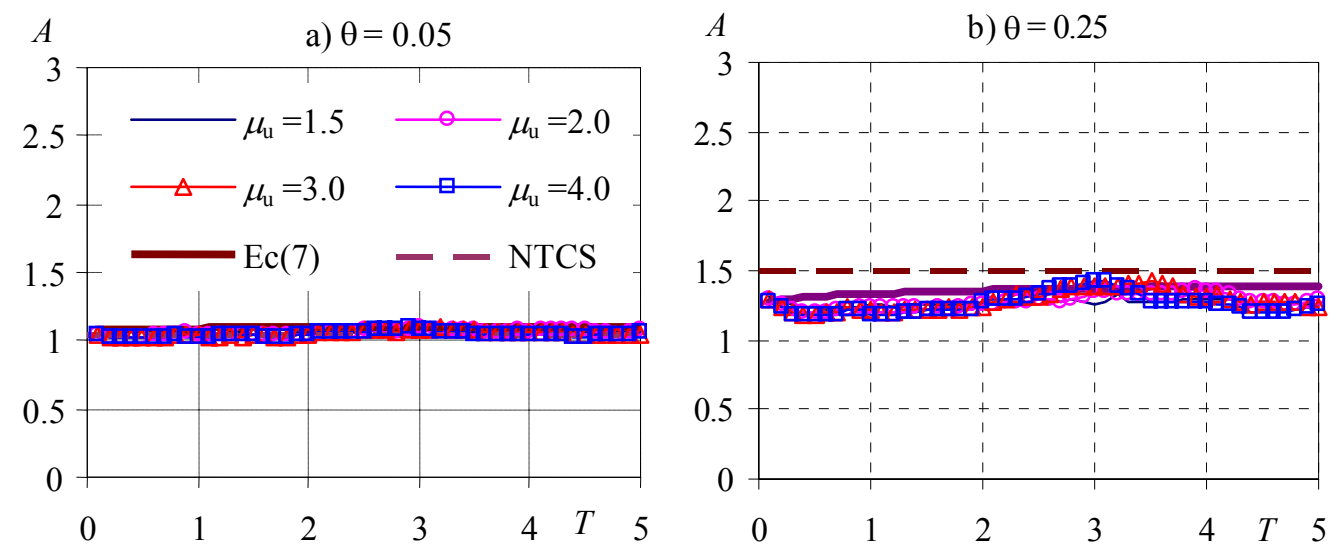

Figura 16. Comparación entre valores medios de $A$ y los valores obtenidos con la ec. 7, Zona de Lomas, Modelo 2
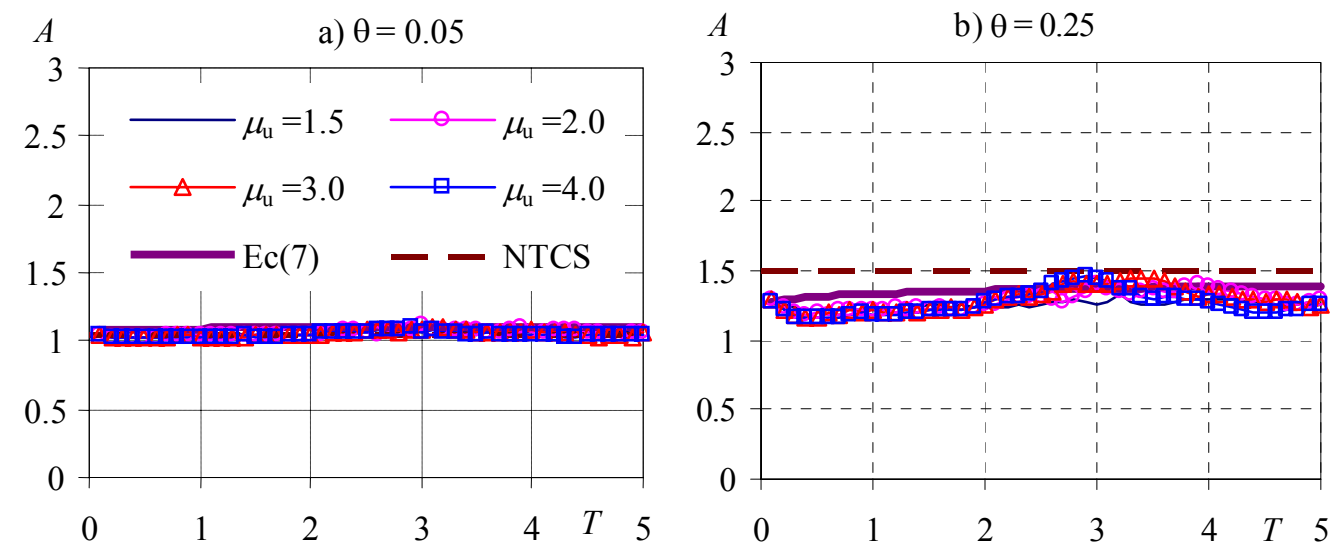

Figura 17. Comparación entre valores medios de $A$ y los valores obtenidos con la ec. 7, Zona de Lomas, Modelo 3
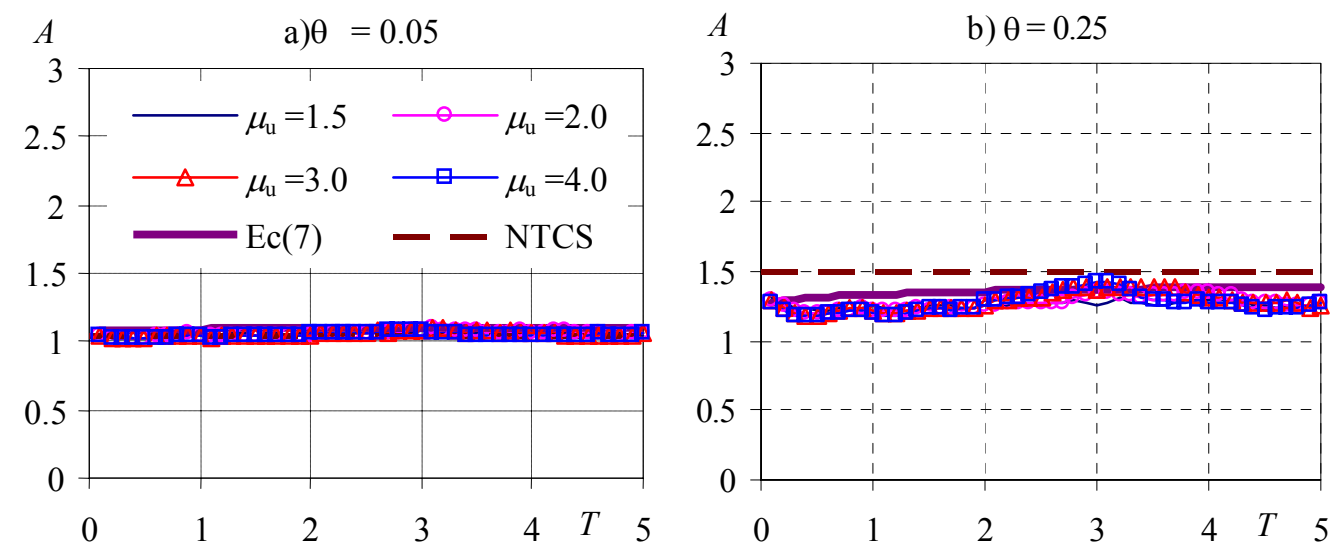

Figura 18. Comparación entre valores medios de $A$ y los valores obtenidos con la ec. 7, Zona de Lomas, Modelo 4 
Las figs. 15 a 18 presentan además los valores de $A$ derivados de las NTCS para marcos dúctiles diseñados para la Zona de Lomas, y que exhiben asimetría en fluencia debido a un desplomo significativo. Puede apreciarse la naturaleza conservadora de las expresiones incluidas en las NTCS, particularmente para valores grandes de $\theta$.

Finalmente, la fig. 19 resume los coeficientes de variación de los valores de $A$ derivados del Modelo 3. Estos valores, que son similares a los obtenidos a partir de los otros comportamientos histeréticos degradantes, confirman la baja variabilidad de los valores de $A$.
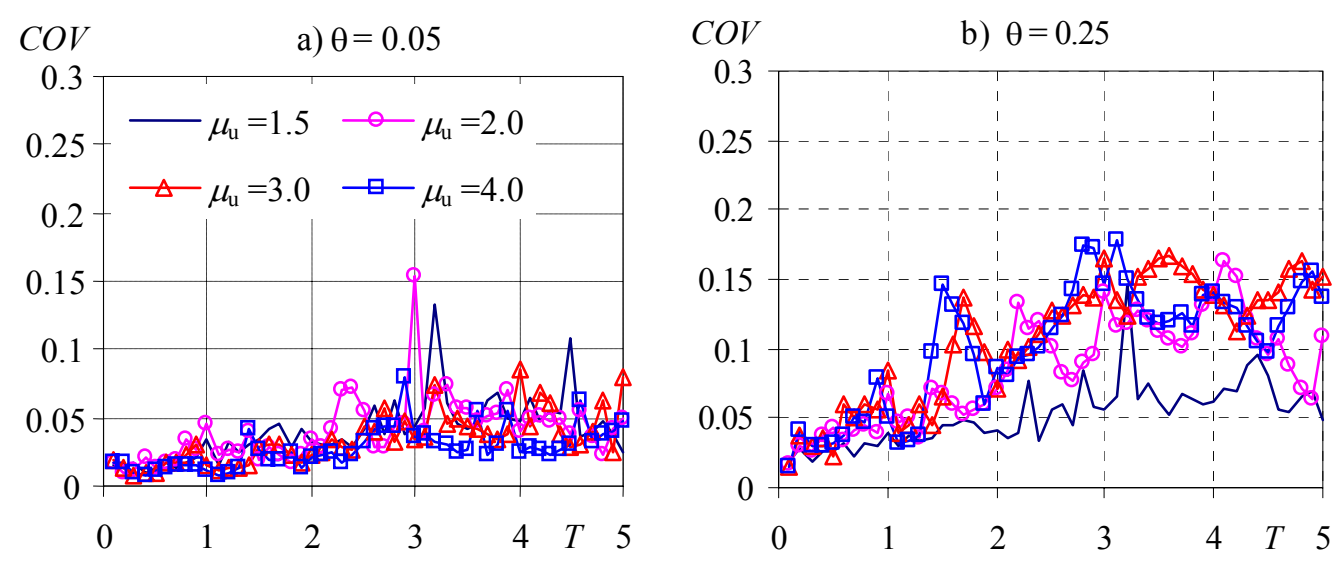

Figura 19. COV de $A$, Zona de Lomas, Modelo 3

\section{CONCLUSIONES}

Una estructura sismorresistente requiere diseñarse para una mayor resistencia lateral conforme crece su asimetría en fluencia. Por tanto, es conveniente definir un factor de amplificación de resistencia que permita el diseño de la resistencia lateral de este tipo de estructuras. En el caso de estructuras con asimetría en fluencia, el criterio utilizado para evaluar su desempeño sísmico influye significativamente en la cuantificación de los factores de amplificación de resistencia. Dada la importancia de la variación de las demandas acumuladas de deformación plástica en sistemas asimétricos, así como las tendencias de plantear métodos de diseño sísmico más racionales, es conveniente evaluar el factor de amplificación de resistencia por medio de índices de daño que hagan consideración explícita de las demandas de energía plástica

A pesar de que los resultados obtenidos por varios investigadores sugieren la necesidad de actualizarlos, los requerimientos incluidos en las NTCS para el diseño de estructuras con asimetría en fluencia permanecen sin cambio desde que fueron introducidos en 1987. Dentro de las deficiencias más importantes de estos requerimientos puede decirse que el factor de amplificación de resistencia estimado conforme a ellos:

- Se establece de manera independiente del periodo fundamental de vibración y del comportamiento histerético de la estructura. Esto a pesar de la fuerte dependencia que $A$ exhibe respecto a dichas características. 
- Es constante para las tres zonas sísmicas consideradas por las NTCS (Zona de Lomas, Zona de Transición y Zona del Lago). Esto a pesar de que el valor de $A$ puede variar considerablemente en función del tipo de suelo donde se desplanta la estructura.

- Cuantifica el nivel de asimetría en fluencia en la estructura exclusivamente a través del desplomo, sin tomar en consideración la resistencia lateral esperada de la estructura. Esto a pesar de que el nivel de asimetría en fluencia solo puede caracterizarse correctamente si se toma en cuenta simultáneamente el desplomo y coeficiente sísmico esperado en la estructura.

En el caso particular de estructuras que exhiben comportamiento similar al elasto-plástico perfecto, las NTCS pueden dar lugar a diseños del lado de la inseguridad. Si se considera que una estructura real difícilmente exhibirá este tipo de comportamiento, puede considerarse que los requerimientos de las NTCS para el diseño de estructuras con asimetría en fluencia suelen ser conservadores con respecto a los definidos para el diseño de estructuras simétricas en fluencia. Sin embargo, dado que el nivel de diseño de estructuras con asimetría en fluencia puede ir desde excesivamente conservador hasta el lado de la inseguridad, es deseable hacer una revisión de los requerimientos de las NTCS.

Las expresiones para estimar el factor de amplificación de resistencia, A, presentadas en este artículo constituyen una base racional para plantear el diseño de la resistencia lateral de estructuras con asimetría en fluencia. Estas expresiones son aplicables a estructuras que no exhiban degradación excesiva ni adelgazamiento del ciclo histerético. Una consideración relevante para establecer requerimientos normativos a partir de las expresiones que aquí se presentan es que el valor de $A$ solo afecta la carga lateral de diseño. Al respecto, parte de la sobrerresistencia que una estructura exhibe ante carga lateral es producto de su sobre-diseño ante carga gravitacional, de tal manera que el incremento en la resistencia lateral de una estructura real puede llegar a ser menor al indicado por el valor de $A$.

Resulta importante recalcar que los resultados reportados en este estudio no son válidos para estructuras que exhiban efectos de segundo orden excesivos, y que no se ha estudiado como afecta la interacción suelo-estructura la demanda de resistencia lateral en sistemas con asimetría en fluencia. Los resultados presentados en este artículo pueden ser utilizados, junto con otros que se desarrollen en el futuro, para plantear, dentro de nuevas versiones de las Normas Técnicas Complementarias para Diseño por Sismo, la resistencia lateral de diseño de estructuras con asimetría en fluencia.

\section{RECONOCIMIENTOS}

Los autores agradecen el apoyo de la Universidad Autónoma Metropolitana, y del Departamento del Distrito Federal. 


\section{REFERENCIAS}

Arroyo, D y A Terán (2004), "Factores de amplificación para sistemas con asimetría en fluencia", Reporte de Investigación 450, Universidad Autónoma Metropolitana, 143 pp., ISBN 970-310273-5.

Cosenza, E, G Manfredi y R Ramasco (1993), "The use of damage functionals in earthquake engineering: a comparison between different methods", Earthquake Engineering and Structural Dynamics, Vol. 22, No. 10, pp. 855-868.

Kunnath, S K, A M Reinhorn y Y J Park (1990), "Analytical modeling of inelastic seismic response of R/C structures", ASCE Journal of Structural Engineering, Vol. 116, No. 4, pp. 9961017.

Gutiérrez, B (2002), "Estudio de sobrerresistencia en estructuras", Proyectos Terminales I y II de Ingeniería Civil, Departamento de Materiales, Universidad Autónoma Metropolitana Azcapotzalco.

Park, Y J y A H Ang (1985), "Mechanistic seismic damage model for reinforced concrete", ASCE Journal of Structural Engineering, Vol. 111, No. 4, pp. 740-757.

Qi, X y J P Moehle (1991), "Displacement design approach for reinforced concrete structures subjected to earthquakes", Reporte No.UCB/EERC-91/02, Universidad de California, Berkeley.

Ruiz, S, E Rosenblueth y R Diederich (1989), "Seismic response of asymmetrically yielding structures”, Earthquake Spectra, Vol. 5 No. 1, pp. 103-112.

Ruiz, S y H Rosas, (1990), "Factor de amplificación de la respuesta de estructuras con resistencia asimétrica", Revista de Ingeniería Sísmica, SMIS, No. 39, pp. 15-26.

Silva, H y O López (2001), "Estudio experimental sobre índices de daño en estructuras de concreto reforzado sujetas a cargas laterales", Memorias, XIII Congreso Nacional de Ingeniería Sísmica, Guadalajara, México, CDROM.

Terán, A, J Carballo y V V Bertero (1993), "Comportamiento no lineal de estructuras inclinadas", Memorias, X Congreso Nacional de Ingeniería Sísmica, Puerto Vallarta, México.

Terán, A, H Juárez y M Frausto (1998), "Efectos de la asimetría en fluencia en el comportamiento sísmico de sistemas estructurales", Revista de Ingeniería Sísmica, SMIS, No. 58, pp. 41-63.

Terán, A (1998), “Características mecánicas y desempeño sísmico de marcos dúctiles de concreto reforzado", Memorias, XI Congreso Nacional de Ingeniería Estructural, Vol. 1, pp. 564-573, Monterrey, México.

Terán, A (2002), "Consideraciones de uso de la energía plástica en el diseño sísmico”, Revista de Ingeniería Sísmica, SMIS, No. 65, pp. 81-110.

Terán, A y J O Jirsa (2003), "Un modelo simple para predecir la ocurrencia de fatiga de bajo número de ciclos", Memorias, XIV Congreso Nacional de Ingeniería Sísmica, León, México, CDROM.

Terán-Gilmore, A (1996), "Performance-based earthquake-resistant design of framed buildings using energy concepts", Tesis de Doctorado, Universidad de California, Berkeley. 
Terán-Gilmore, A, H Juárez y M Frausto (2000), "On the dynamic response of regular structures exhibiting tilt", Earthquake Engineering and Structural Dynamics, Vol. 29, No. 9, pp. 13431374.

Tung, A, J N Wang, A S Kiremidjian y E Kavazanjian (1992), "Statistical parameters of AM and PSD functions for the generation of site-specific strong ground motions", Memorias, X Congreso Mundial de Ingeniería Sísmica, Vol. 2, pp. 867-872, Madrid, España.

Tso, W K, T J Zhu y A C Heidebrecht (1993), "Seismic energy demands on reinforced concrete moment-resisting frames", Earthquake Engineering and Structural Dynamics, Vol. 22, No. 6, pp. 533-545.

Williams, M S y R G Sexsmith (1997), "Seismic assessment of concrete bridges using inelastic damage analysis”, Engineering Structures, Vol. 19, No. 3, pp. 208-216. 